\title{
Destructive interference in transfer rates by coherent coupling to a remote reservoir
}

\author{
U Schröter and E Scheer \\ University of Konstanz, FB Physik, Universitätsstraße 10, 78464 Konstanz, Germany \\ E-mail: Ursula Schroeter@uni-konstanz.de
}

\begin{abstract}
Transport through two point-contacts in series, even with arbitrarily high transmissions, can be modeled with rate equations if the rates are deduced from the quantum mechanical current formula, which thus accounts for interferences on the wavefunction level. An expression using Green's functions giving the current through a single contact is valid for evaluating the charging rates of the enclosed island in the double-junction system, even in the case of coherent coupling all over the island from lead to lead. We demonstrate that these current rates for each junction can be rewritten as 2-products of the so-called transfer Green functions, maintaining formal analogy to the single-junction case. On the one hand this facilitates numerical calculations. On the other hand, the form of the rate terms reveals how besides direct coupling across one junction effective coupling between the involved and the remote lead contributes to transfers across the regarded junction and how coherent loss from the island across the other junction diminishes the rate. It is further explained that for the double junction in the case of coherent coupling between both contacts direct lead-to-lead transport adds current contributions; however, interferences are expected to reduce the net current as compared to incoherent mutual influence via the island charging only. Crossed Andreev reflection in the superconducting state-within the premises of our model—cannot surpass other transport processes such as to cause extra steps in current-voltage curves or negative differential conductance.
\end{abstract}

PACS numbers: 72.10.Bg, 05.60.Gg, 02.90.+p

\section{Introduction}

Transport across an island of sufficiently small capacitance at low temperature usually exhibits Coulomb blockade (CB) [1, 2], even if in contrast to tiny quantum dots with only few discrete 


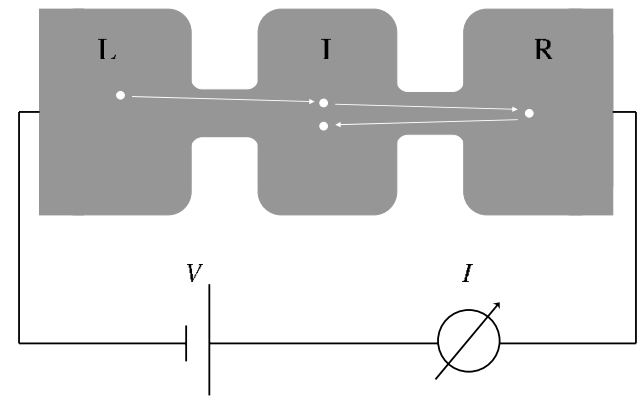

Figure 1. A series of two point contacts with an island (I) between the left (L) and the right (R) lead. A transport process is sketched as an example.

electron states the level spacing is negligible, which is the usual case for metallic islands. Charging then does not influence the form of the density of states (DOS), but simply shifts the DOS together with the Fermi level due to the electrostatic potential. The single-electron transistor [3,4] with tunnel junctions from the island to the leads is a well-studied device in the normal as well as in the superconducting state. Taking into account sequential and co-tunneling - in the superconducting state also including Andreev reflection and Cooper-pair tunneling [5-7] — up to some order suffices to model setups with low-transmission junctions. For contacts accommodating transport channels with arbitrary values of the transmission in the range $0 \ldots 1$ (in units of the conductance quantum $\mathcal{G}_{0}=e^{2} / h$ ), throughputs of multiple reflection processes can no longer independently be added and the current must be calculated on a fully quantum-mechanical basis. Such point contacts can, for example, be arranged with mechanically controllable break-junctions $[8,9]$. In a setup with an island coherence in transport through both junctions or multiple reflections extending over both should be considered. Investigating transport, especially in the superconducting state, through a contact series with one or both junction transmissions clearly out of the tunnel regime is of interest for the following aspects: multiple Andreev reflection (MAR) effectively means transport in multiple-charge packages. It is a subject under study, whether with an island CB prevents MAR in a junction that would exhibit it as a stand-alone device [9]. Modeling is further important to deduce signs in current-voltage characteristics by which to distinguish transport maintaining coherence across the island from incoherent coupling between the two junctions. Our conclusion will, however, be that an ambiguity will hardly be removed.

The premises of our model are the following: the island, left and right leads (figure 1) are bulk reservoirs with the same DOS (assume same material for all three). There is an energy-independent coupling amplitude $t_{L}$ across the left junction and an alike $t_{R}$ for the right junction. (We restrict the problem to one transport channel per junction here ${ }^{1}$.) Coherence is allowed in the sense that a transfer across one junction can be followed by one across the other without relaxation on the island. (In the relaxed state we assume zero-temperature occupation of the DOS.) The purpose of nevertheless treating the island as a bulk reservoir (like the leads) is to also allow transport processes starting on the island in a relaxed state or ending there to relax the charge carrier. (For simplicity we further assume that coherence of transfers through different junctions is as strong as that of successive transfers across the same junction. Later mixing our incoherent and coherent ansatzes to model partial coherence across the island is a conceivable idea.) The varying electrostatic potential of the island with charging

1 A generalization of our so-called coherent model to several channels per junction is under work. 
is taken into account. Assuming a (quasi-particle) density of states-or better a corresponding Green's function $g$-for the reservoirs and a single-hopping amplitude $t$ for each junction in the superconducting state describes electron and hole transport including Andreev reflection [13]. Cooper-pairs would have to be added as an independent transport mechanism only intermingling with quasi-particle transfers via the island charging. Cooper-pairs are not yet included in our calculations. In the normal state charge carriers are electrons (the model requires electrons and missing electrons $=$ holes, though).

The following sections will concentrate on the couplings across the two junctions. However, keeping in mind that the leads are connected to a voltage source, to deal with the non-equilibrium transport problem, Green's functions are needed on the original site bases L, I and R. We are not looking for eigenstates of a closed system. Although the application discussed is quantum transport through a point-contact series, the main emphasize of this work is on formal properties of Green's functions, and most relations for them are given in detail. The general problem of three reservoirs, one of which is coherently coupled to the other two, might also be found in totally different applications.

\section{Three-site Green's and transfer functions}

Corresponding to the three sites L, I and R the Hamiltonian can be written in a matrix form as

$$
H=\left(\begin{array}{ccc}
H_{L L} & \sigma_{L I} & 0 \\
\sigma_{I L} & H_{I I} & \sigma_{I R} \\
0 & \sigma_{R I} & H_{R R}
\end{array}\right) \text { acting on the wavefunction } \Psi=\left(\begin{array}{c}
\Psi_{L} \\
\Psi_{I} \\
\Psi_{R}
\end{array}\right) ;
$$

$H_{L L}, H_{I I}$ and $H_{R R}$ are the Hamiltonians of bulk reservoirs as if no connections via the junctions existed. $g_{L L}, g_{I I}$ and $g_{R R}$ denote the corresponding Green's functions [12]. $\sigma$ are the couplings between sites established by the junctions. Then the advanced and retarded Green's functions of the complete system satisfy the Dyson equation:

$$
\begin{aligned}
\left(\begin{array}{ccc}
G_{L L} & G_{L I} & G_{L R} \\
G_{I L} & G_{I I} & G_{I R} \\
G_{R L} & G_{R I} & G_{R R}
\end{array}\right)=\left(\begin{array}{ccc}
g_{L L} & 0 & 0 \\
0 & g_{I I} & 0 \\
0 & 0 & g_{R R}
\end{array}\right) \\
+\left(\begin{array}{ccc}
g_{L L} & 0 & 0 \\
0 & g_{I I} & 0 \\
0 & 0 & g_{R R}
\end{array}\right)\left(\begin{array}{ccc}
0 & \sigma_{L I} & 0 \\
\sigma_{I L} & 0 & \sigma_{I R} \\
0 & \sigma_{R I} & 0
\end{array}\right)\left(\begin{array}{lll}
G_{L L} & G_{L I} & G_{L R} \\
G_{I L} & G_{I I} & G_{I R} \\
G_{R L} & G_{R I} & G_{R R}
\end{array}\right) .
\end{aligned}
$$

For the transport problem it is of advantage to work with $T$ [11] instead of $G$ defined through $G \sigma=g T$ or equivalently $\sigma G=T g$ (without subscripts letters mean the respective $3 \times 3$ matrix). For clarity, these equations are now written out as full matrices because care must be taken that for some components there is a sum of products on the left side:

$$
\begin{aligned}
& \left(\begin{array}{ccc}
G_{L I} \sigma_{I L} & G_{L L} \sigma_{L I}+G_{L R} \sigma_{R I} & G_{L I} \sigma_{I R} \\
G_{I I} \sigma_{I L} & G_{I L} \sigma_{L I}+G_{I R} \sigma_{R I} & G_{I I} \sigma_{I R} \\
G_{R I} \sigma_{I L} & G_{R L} \sigma_{L I}+G_{R R} \sigma_{R I} & G_{R I} \sigma_{I R}
\end{array}\right)=\left(\begin{array}{ccc}
g_{L L} T_{L L} & g_{L L} T_{L I} & g_{L L} T_{L R} \\
g_{I I} T_{I L} & g_{I I} T_{I I} & g_{I I} T_{I R} \\
g_{R R} T_{R L} & g_{R R} T_{R I} & g_{R R} T_{R R}
\end{array}\right) \\
& \left(\begin{array}{ccc}
\sigma_{L I} G_{I L} & \sigma_{L I} G_{I I} & \sigma_{L I} G_{I R} \\
\sigma_{I L} G_{L L}+\sigma_{I R} G_{R L} & \sigma_{I L} G_{L I}+\sigma_{I R} G_{R I} & \sigma_{I L} G_{L R}+\sigma_{I R} G_{R R} \\
\sigma_{R I} G_{I L} & \sigma_{R I} G_{I I} & \sigma_{R I} G_{I R}
\end{array}\right) \\
& =\left(\begin{array}{lll}
T_{L L} g_{L L} & T_{L I} g_{I I} & T_{L R} g_{R R} \\
T_{I L} g_{L L} & T_{I I} g_{I I} & T_{I R} g_{R R} \\
T_{R L} g_{L L} & T_{R I} g_{I I} & T_{R R} g_{R R}
\end{array}\right) ;
\end{aligned}
$$


$T$ obeys the Dyson equation:

$$
\begin{aligned}
\left(\begin{array}{ccc}
T_{L L} & T_{L I} & T_{L R} \\
T_{I L} & T_{I I} & T_{I R} \\
T_{R L} & T_{R I} & T_{R R}
\end{array}\right)=\left(\begin{array}{ccc}
0 & \sigma_{L I} & 0 \\
\sigma_{I L} & 0 & \sigma_{I R} \\
0 & \sigma_{R I} & 0
\end{array}\right) \\
+\left(\begin{array}{ccc}
0 & \sigma_{L I} & 0 \\
\sigma_{I L} & 0 & \sigma_{I R} \\
0 & \sigma_{R I} & 0
\end{array}\right)\left(\begin{array}{ccc}
g_{L L} & 0 & 0 \\
0 & g_{I I} & 0 \\
0 & 0 & g_{R R}
\end{array}\right)\left(\begin{array}{ccc}
T_{L L} & T_{L I} & T_{L R} \\
T_{I L} & T_{I I} & T_{I R} \\
T_{R L} & T_{R I} & T_{R R}
\end{array}\right)
\end{aligned}
$$

Note that some components of (5) will have two terms of the form $\sigma g T$ on the right side. With full coherence a process from the island I can start off in any direction, no matter where it is going to end. We note all nine component equations here and also the results from the complementary Dyson equation $T=\sigma+T g \sigma$ :

$$
\begin{aligned}
& T_{L L}=\sigma_{L I} g_{I I} T_{I L}=T_{L I} g_{I I} \sigma_{I L} \\
& T_{L I}=\sigma_{L I}+\sigma_{L I} g_{I I} T_{I I}=\sigma_{L I}+T_{L L} g_{L L} \sigma_{L I}+T_{L R} g_{R R} \sigma_{R I} \\
& T_{L R}=\sigma_{L I} g_{I I} T_{I R}=T_{L I} g_{I I} \sigma_{I R} \\
& T_{I L}=\sigma_{I L}+\sigma_{I L} g_{L L} T_{L L}+\sigma_{I R} g_{R R} T_{R L}=\sigma_{I L}+T_{I I} g_{I I} \sigma_{I L} \\
& T_{I I}=\sigma_{I L} g_{L L} T_{L I}+\sigma_{I R} g_{R R} T_{R I}=T_{I L} g_{L L} \sigma_{L I}+T_{I R} g_{R R} \sigma_{R I} \\
& T_{I R}=\sigma_{I R}+\sigma_{I L} g_{L L} T_{L R}+\sigma_{I R} g_{R R} T_{R R}=\sigma_{I R}+T_{I I} g_{I I} \sigma_{I R} \\
& T_{R L}=\sigma_{R I} g_{I I} T_{I L}=T_{R I} g_{I I} \sigma_{I L} \\
& T_{R I}=\sigma_{R I}+\sigma_{R I} g_{I I} T_{I I}=\sigma_{R I}+T_{R L} g_{L L} \sigma_{L I}+T_{R R} g_{R R} \sigma_{R I} \\
& T_{R R}=\sigma_{R I} g_{I I} T_{I R}=T_{R I} g_{I I} \sigma_{I R} .
\end{aligned}
$$

It does not matter whether $T$ is obtained by a direct solution of (5) or the two-step procedure described in [11]; it is the function satisfying (3), (4) and (5), relations which will be used in the following manipulations. As a further preparation the constituting equation for $G^{+-}$, namely $G^{+-}=\left[\mathbf{1}+G^{r} \sigma\right] g^{+-}\left[\mathbf{1}+\sigma G^{a}\right]$, has to be multiplied out in three-site space:

$$
\begin{aligned}
& \left(\begin{array}{ccc}
G_{L L}^{+-} & G_{L I}^{+-} & G_{L R}^{+-} \\
G_{I L}^{+-} & G_{I I}^{+-} & G_{I R}^{+-} \\
G_{R L}^{+-} & G_{R I}^{+-} & G_{R R}^{+-}
\end{array}\right)=\left[\left(\begin{array}{ccc}
1 & 0 & 0 \\
0 & 1 & 0 \\
0 & 0 & 1
\end{array}\right)+\left(\begin{array}{ccc}
G_{L L}^{r} & G_{L I}^{r} & G_{L R}^{r} \\
G_{I L}^{r} & G_{I I}^{r} & G_{I R}^{r} \\
G_{R L}^{r} & G_{R I}^{r} & G_{R R}^{r}
\end{array}\right)\left(\begin{array}{ccc}
0 & \sigma_{L I} & 0 \\
\sigma_{I L} & 0 & \sigma_{I R} \\
0 & \sigma_{R I} & 0
\end{array}\right)\right] \\
& \cdot\left(\begin{array}{ccc}
g_{L L}^{+-} & 0 & 0 \\
0 & g_{I I}^{+-} & 0 \\
0 & 0 & g_{R R}^{+-}
\end{array}\right) \cdot\left[\left(\begin{array}{ccc}
1 & 0 & 0 \\
0 & 1 & 0 \\
0 & 0 & 1
\end{array}\right)+\left(\begin{array}{ccc}
0 & \sigma_{L I} & 0 \\
\sigma_{I L} & 0 & \sigma_{I R} \\
0 & \sigma_{R I} & 0
\end{array}\right)\left(\begin{array}{ccc}
G_{L L}^{a} & G_{L I}^{a} & G_{L R}^{a} \\
G_{I L}^{a} & G_{I I}^{a} & G_{I R}^{a} \\
G_{R L}^{a} & G_{R I}^{a} & G_{R R}^{a}
\end{array}\right)\right] .
\end{aligned}
$$

In an exemplary way we here give the results for the two components needed later:

$$
\begin{aligned}
& G_{I R}^{+-}=G_{I I}^{r} \sigma_{I L} g_{L L}^{+-} \sigma_{L I} G_{I R}^{a}+G_{I I}^{r} \sigma_{I R} g_{R R}^{+-}\left(1+\sigma_{R I} G_{I R}^{a}\right) \\
&+\left(1+G_{I L}^{r} \sigma_{L I}+G_{I R}^{r} \sigma_{R I}\right) g_{I I}^{+-}\left(\sigma_{I L} G_{L R}^{a}+\sigma_{I R} G_{R R}^{a}\right) \\
& G_{R I}^{+-}=G_{R I}^{r} \sigma_{I L} g_{L L}^{+-} \sigma_{L I} G_{I I}^{a}+\left(1+G_{R I}^{r} \sigma_{I R}\right) g_{R R}^{+-} \sigma_{R I} G_{I I}^{a} \\
&+\left(G_{R L}^{r} \sigma_{L I}+G_{R R}^{r} \sigma_{R I}\right) g_{I I}^{+-}\left(1+\sigma_{I L} G_{L I}^{a}+\sigma_{I R} G_{R I}^{a}\right)
\end{aligned}
$$

Remark that a component of $G^{+-}$also contains contributions with the $g^{+-}$at the site(s) different from its own indices. The given $G_{I R / R I}^{+-}$each have a term with $g_{L L}^{+-}$. Primarily, 


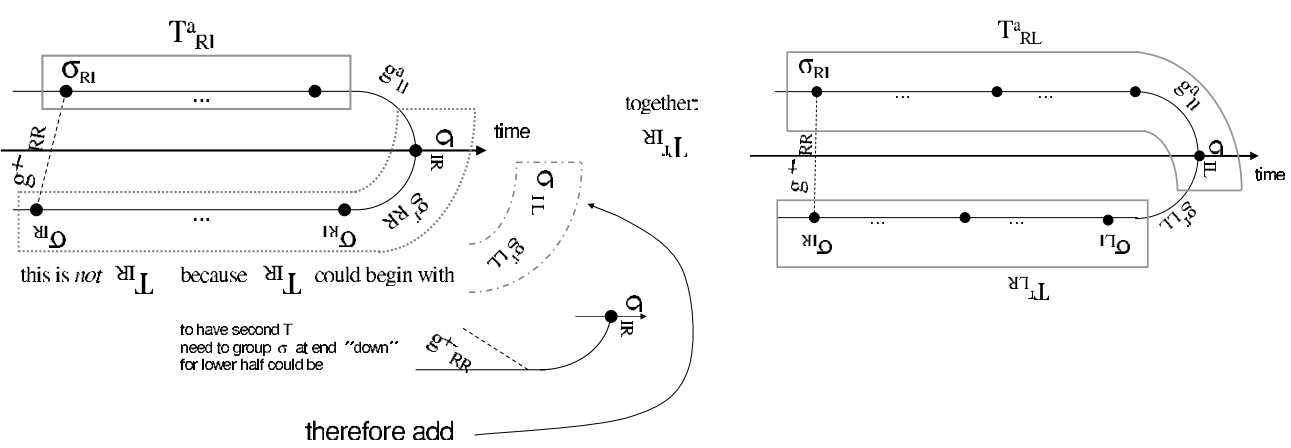

Figure 2. Illustrating the deduction (11) on the Keldysh contour.

$g, G$ and $T$ are functions of two time arguments; the $\sigma$ describing the hopping between sites have a time-dependent phase factor $[10,12,13]$. All expressions and equations written as multiplications mean convolution-like integration over inner time arguments ( $\tau$ will be used for time variables.) When using Fourier representations, all the given relations become mere algebraic equations with products of $g, G$ and $T$ as functions of a single frequency argument each.

\section{Charging rates}

It will be necessary to evaluate expressions like $G_{R I}^{+-} \sigma_{I R}$. Inserting (9) for $G_{R I}^{+-}$and then using (3) and (4) to replace $G$ by $T$ we obtain

$$
\begin{aligned}
G_{R I}^{+-} \sigma_{I R}= & g_{R R}^{r} T_{R L}^{r} g_{L L}^{+-} T_{L I}^{a} g_{I I}^{a} \sigma_{I R}+\left(1+g_{R R}^{r} T_{R R}^{r}\right) g_{R R}^{+-} T_{R I}^{a} g_{I I}^{a} \sigma_{I R} \\
& +g_{R R}^{r} T_{R I}^{r} g_{I I}^{+-}\left(1+T_{I I}^{a} g_{I I}^{a}\right) \sigma_{I R} \\
= & g_{R R}^{r} T_{R L}^{r} g_{L L}^{+-} T_{L R}^{a}+\left(1+g_{R R}^{r} T_{R R}^{r}\right) g_{R R}^{+-} T_{R R}^{a}+g_{R R}^{r} T_{R I}^{r} g_{I I}^{+-} T_{I R}^{a} .
\end{aligned}
$$

Some relations out of (6) have been used to get the last line. $T_{R I} g_{I I} \sigma_{I R}$ in the second term need not have been contracted into $T_{R R}$. This term shall be treated differently now. Equation (10) is written in components in site space, but each $T, g$ and $\sigma$ still is a $2 \times 2$ matrix in $e h$-space. Nevertheless, the trace of $G_{R I}^{+-} \sigma_{I R}$ in $e h$-space will finally be the only required quantity. As cyclic permutation in matrix multiplication leaves the trace unchanged, $\sigma_{I R}$ can be brought in front. After (6), $\sigma_{I R}+\sigma_{I R} g_{R R} T_{R R}$, however, to be contracted into $T_{I R}$ misses the term $\sigma_{I L} g_{L L} T_{L R}$. This is therefore added and subtracted, and $\sigma_{I L}$ of the subtracted again brought to the end, where $T_{R I} g_{I I} \sigma_{I L}$ then simplifies to $T_{R L}$ (see also figure 2):

$$
\begin{aligned}
\operatorname{Tr}\left(\sigma_{I R}(1+\right. & \left.\left.g_{R R}^{r} T_{R R}^{r}\right) g_{R R}^{+-} T_{R I}^{a} g_{I I}^{a}\right) \\
& =\operatorname{Tr}\left(\left(\sigma_{I R}+\sigma_{I L} g_{L L}^{r} T_{L R}^{r}+\sigma_{I R} g_{R R}^{r} T_{R R}^{r}\right) g_{R R}^{+-} T_{R I}^{a} g_{I I}^{a}-g_{L L}^{r} T_{L R}^{r} g_{R R}^{+-} T_{R I}^{a} g_{I I}^{a} \sigma_{I L}\right) \\
& =\operatorname{Tr}\left(T_{I R}^{r} g_{R R}^{+-} T_{R I}^{a} g_{I I}^{a}-g_{L L}^{r} T_{L R}^{r} g_{R R}^{+-} T_{R L}^{a}\right)
\end{aligned}
$$

We now explain why (10) and similar expressions give the transport rates we need and how these get used in a master equation. Even with coherent transport through both junctions, the current (we skip factor $e$ for unit charge) when evaluated as seen from the left side 
[12] from

$$
\left\langle\frac{\mathrm{d} \rho_{L}}{\mathrm{~d} \tau}\right\rangle=\left\langle[\left(\begin{array}{ccc}
H_{L L} & \sigma_{L I} & 0 \\
\sigma_{I L} & H_{I I} & \sigma_{I R} \\
0 & \sigma_{R I} & H_{R R}
\end{array}\right), \underbrace{\left(\begin{array}{ccc}
1 & 0 & 0 \\
0 & 0 & 0 \\
0 & 0 & 0
\end{array}\right)}_{\rho_{L}}]\right\rangle=\left\langle\left(\begin{array}{ccc}
0 & -\sigma_{L I} & 0 \\
\sigma_{I L} & 0 & 0 \\
0 & 0 & 0
\end{array}\right)\right\rangle
$$

comes out as $\operatorname{Tr}\left(G_{L I}^{+-} \sigma_{I L}-\sigma_{L I} G_{I L}^{+-}\right)$, the identical expression to the single junction [13] (with the right side named I instead of $\mathrm{R}$ ). There are no additional terms like $\sigma_{L I} \sigma_{I R} G_{R L}^{+-}$, although $G_{R L}^{+-}$exists. Nevertheless, in contrast to the incoherent model [10] interaction to the Rreservoir is now contained in $G_{L I / I L}^{+-} \cdot\left\langle\frac{\mathrm{d} \rho_{R}}{\mathrm{~d} \tau}\right\rangle$ gives an analogous result, of course. A stationary state corresponding to a long-time measurement implies that the current (dc) is the same through both junctions. One can prove that $\operatorname{Tr}\left(G_{R I}^{+-} \sigma_{I R}-\sigma_{R I} G_{I R}^{+-}\right)=2 \operatorname{Re}\left\{\operatorname{Tr}\left(G_{R I}^{+-} \sigma_{I R}\right)\right\}$. Furthermore, in the same sense that an expectation value like in (12) means taking the trace in site space [12], in the superconducting state with electron and hole quasi-particles the trace also has to be taken in electron-hole space. Calculating the time derivative of the charge on the island as the commutator with the Hamiltonian, using the scheme (12) with $\rho_{I}=\left(\begin{array}{lll}0 & 0 & 0 \\ 0 & 1 & 0 \\ 0 & 0 & 0\end{array}\right)$ yields

$$
\left\langle\frac{\mathrm{d} \rho_{I}}{\mathrm{~d} \tau}\right\rangle=\operatorname{Tr}\left(\sigma_{L I} G_{I L}^{+-}-G_{L I}^{+-} \sigma_{I L}+\sigma_{R I} G_{I R}^{+-}-G_{R I}^{+-} \sigma_{I R}\right)
$$

Naturally, transport rates through both junctions add for the island. Here, with in order to model coherent interaction between the left and right junctions considering the whole system in the quantum-mechanical ansatz (1), (13) comes as a direct result of Ehrenfest's theorem. In the incoherent model [10] adding charging rates from both junctions for the island is done as part of setting up a classical rate equation. The result is formally identical to (13), the $G^{+-}$ however stemming from solving respective $2 \times 2$ Dyson equations for each junction separately.

The island will change its potential with charging and at least for all cases of nonidentical junction transmissions this effect is mandatory for the establishment of a stationary dc current flow. Different island charge states behave like different reservoirs [10]. This picture is the same for the incoherent and the coherent model. Setting up the rate equation $\overrightarrow{\vec{P}}=R \cdot \vec{P}=0$ and solving for the charge-state probabilities $\vec{P}=\left(P_{n}\right)$ therefore stays the same procedure. As explained in [10], doing manipulations like (10) with island charge indices requires treating $e e$ - and $h h$-parts of $G_{R I}^{+-} \sigma_{I R}$ separately, and if, for example, there is $\sigma_{I R}^{n+1 n}$ for an electron hopping, moving this $\sigma$ from the end to the beginning of a product changes the outer charge index from $n$ to $n+1$. An inner charge index $n_{2}$ is summed over in all products like, for example, $g_{R R}^{r ; n n} T_{R L}^{r ; n n_{2}} g_{L L}^{+-, n_{2} n_{2}} T_{L R}^{a, n_{2} n}$. In (13) considering the transfers across the left junction that increase the island charge to $n+1$ compared to it being $n$ when the hopping quasi-particle is in the left lead before or after, picks the contributions with $\sigma$ of electron type. In $\left(\sigma_{L I}^{n n+1} G_{I L}^{+-, n+1 n}\right)_{e e}-\left(G_{L I}^{+-, n n+1} \sigma_{I L}^{n+1 n}\right)_{e e}$ the second term including the minus sign with the charge indices reversed together with the site indices indeed turns out to be the complex conjugate of the first term. Analogously, there are the hole transfers $\left(\sigma_{L I}^{n+1 n} G_{I L}^{+-, n n+1}\right)_{h h}-\left(G_{L I}^{+-, n+1 n} \sigma_{I L}^{n n+1}\right)_{h h}$ that also change the island charge between $n$ and $n+1$. These terms are to be taken with opposite sign for the opposite charge of holes as compared to electrons. The electron and hole contributions have to be taken together [10] before determining where the net result is to be put in the rate matrix. If it is positive it is added at a place meaning that transfers across the left junction will increase the island charge to $n+1$ if the island is found in state $n$. If it is negative its absolute value is added to the entry meaning decrease to $n$ will happen if the island is found in state $n+1$. Only one way will 
processes take place across a junction (we regarded the left here), with a frequency further depending on either $P(n)$ or $P(n+1)$. Our rates already constitute net values according to backreflections across a channel playing between the same $n$ and $n+1$. A product with a $\sigma$ with I associated with $n$ involves the DOS of the island state $n$ as if that remained static during the single transfer. The same holds for the $n+1$ island state DOS for the terms where $n+1$ is with I on $\sigma$. L (or R) has fixed Fermi level, its DOS is independent of the island charge. Counterbalancing the $n \rightarrow n+1$ rate through a channel by the $n+1 \rightarrow n$ rate as well as taking electrons and holes together before entering the value into the rate matrix in our model has precisely the effect of ensuring enough energy to be gained by the electron or hole passing the voltage over a junction to change the island potential with the transfer. In decharging it also has to be guaranteed that the island DOS does not shift so much during transfer as to prevent the off-coming charge from finding an appropriate level in a lead. As a marginal consistency check, we briefly mention that with setting up the rate matrix in the described way the correct behavior in current-voltage characteristics is reproduced in the well-known limit of low transmission and vanishing superconductor gap. In the sense whether there will be a rate to bring the island charge up from $n$ to $n+1$ or down from $n+1$ to $n$, transfers across the right junction are independent of those through the left. Even with coherent coupling across the island the transport channels from both junctions do not merge into an effective one from lead to lead, but the remaining two channels are coupled together by the island. The two parts in (13), by the way, represent expectation values on their own, namely $-\left\langle\mathrm{d} \rho_{L} / \mathrm{d} \tau\right\rangle$ and $-\left\langle\mathrm{d} \rho_{R} / \mathrm{d} \tau\right\rangle$. Therefore despite the ansatz with hoppings through both junctions in one Hamiltonian, the rate equation is set up from transfers across the left and right junctions treated separately in the coherent model [14] in the same way as in the incoherent model [10].

There is no problem in taking the island charge as a good quantum number [10]. However, there may still be some subtlety about the kind of simultaneousness of transport processes allowed. Each single hopping $\sigma$ counted in terms à la (13) can be part of a multiple (Andreev) reflection. The preceding and following hoppings in these processes may be only virtual. The island does not actually need to be charged to the other states involved apart from $n$ and $n+1$. Consequently, from a chain of single hoppings in a MAR each one need not really happen exactly as often as all others. For understanding one should think of the $n \leftrightarrow n+1$ charging rate as expanded into the sum of the rates for all MAR processes that contain a single hopping between states $n$ and $n+1$ through a junction. In determining whether this rate-or again think of each process contribution in it separately-is positive, zero or negative, the possibility of each such MAR process around the single $n \leftrightarrow n+1$ hopping is checked instantaneously, that is with the MAR process determining all island states before and after exclusively by its own. All these energy levels have an influence on the calculated amplitudes of that rate contribution. Evaluating the amplitude of a charging step in a process as if exclusively this process were happening in the system is based on the physical conception to keep coherence in every way unbroken during the process ${ }^{2}$. For simplicity we just spoke of rate contributions from different processes; section 4 will, however, illustrate that, with respect to multiple reflection processes, at the basis amplitudes are evaluated and then interference between processes is included in charging rates. Due to the effective decomposition of multiple (Andreev) reflection processes into single steps for evaluating charging rates, the rate matrix is quite simple with entries only for transitions $n \rightarrow n-1, n \rightarrow n, n \rightarrow n+1$.

The actual evaluation of terms such as (10) and (11) is performed using Fourier representations $[10,13,14] . \quad g^{r / a /+-}\left(\tau, \tau^{\prime}\right)=\frac{1}{2 \pi} \int \mathrm{d} \omega \mathrm{e}^{-\mathrm{i} \omega \tau} \mathrm{e}^{\mathrm{i} \omega \tau^{\prime}} g^{r / a /+-}(\omega)$ with $g(\omega)$

2 Allowing other transport processes change the island charge during the regarded MAR would also present an ansatz not principally to be rejected, however, be based on another conception as to how actions of different quasi-particles from a reservoir are linked together. This idea is planned to be developed elsewhere. 
analytically given functions $[12,13]$, the same for all reservoirs. $g$ cannot change the island charge, it is always $g^{n n}$ :

$$
\begin{aligned}
T_{R I}^{r ; n_{1} n_{2}}\left(\tau, \tau^{\prime}\right) & =\sum_{k} \sum_{p} \int \frac{\mathrm{d} \omega}{2 \pi} \mathrm{e}^{-\mathrm{i} \omega \tau} \mathrm{e}^{-\mathrm{i} k A \tau} \mathrm{e}^{-\mathrm{i} m B \tau} \mathrm{e}^{-\mathrm{i} p C \tau} \mathrm{e}^{\mathrm{i} \omega \tau^{\prime}} T_{R I, k p}^{r ; n_{1} n_{2}}(\omega) \\
& =\sum_{k} \sum_{p} \int \frac{\mathrm{d} \omega}{2 \pi} \mathrm{e}^{-\mathrm{i} \omega \tau} \mathrm{e}^{\mathrm{i} k A \tau^{\prime}} \mathrm{e}^{\mathrm{i} m B \tau^{\prime}} \mathrm{e}^{\mathrm{i} p C \tau^{\prime}} \mathrm{e}^{\mathrm{i} \omega \tau^{\prime}}\left(T_{I R, k p}^{a ; n_{2} n_{1}}(\omega)\right)^{\dagger}
\end{aligned}
$$

Expressions for advanced $T$ and other space indices are analogous. The second line indicating the link between $T_{i j}^{r / a ; n_{1} n_{2}}$ and $T_{j i}^{a / r ; n_{2} n_{1}}$ is of practical importance. The dagger $\dagger$ means complex conjugation as well as for the superconducting state transposition of $2 \times 2$ matrices in electron-hole space with giving an extra minus sign to the off-diagonal $e h$ - and $h e$-components. $k$ and $p$ run over all possible integer combinations with the restriction that $n_{2}-n_{1}=k+p$. (We explicitly write the double sum, because we intend to take $n_{2}$ as the dependent variable later on.) $A$ and $C$ designate the voltage drops multiplied by $e$ and divided by $\hbar$ over the left and the right junctions, respectively, in case of a neutral island. This division of the voltage applied between the left and the right lead is determined by the ratio of the capacitances associated with the two junctions. The effect of a gate voltage in case of an additional electrode only capacitively coupled to the island can be included [10]. $B$ denotes twice the island charging energy divided by $\hbar$ and the integer $m$ is a function of $k, p$ and $n_{1}$. Using (11) in (10) means that $G_{R I}^{+-, n n+1} \sigma_{I R}^{n+1 n}$ consists of four terms. We use the Fourier representation of the kind (15) for $T_{R L}^{r}$ and the kind (14) for $T_{L R}^{a}$ in $g_{R R}^{r, n n} T_{R L}^{r, n n_{2}} g_{L L}^{+-, n_{2} n_{2}} T_{L R}^{a, n_{2} n}$, (15) for $T_{R I}^{r}$ and (14) for $T_{I R}^{a}$ in $g_{R R}^{r, n n} T_{R I}^{r, n n_{2}} g_{I I}^{+-, n_{2} n_{2}} T_{I R}^{a, n_{2} n}$, (14) for $T_{I R}^{r}$ and (15) for $T_{R I}^{a}$ in $T_{I R}^{r, n+1 n_{2}} g_{R R}^{+-; n_{2} n_{2}} T_{R I}^{a, n_{2} n+1} g_{I I}^{a, n+1 n+1}$, (14) for $T_{L R}^{r}$ and (15) for $T_{R L}^{a}$ in $g_{L L}^{r, n n} T_{L R}^{r, n} n_{2} g_{R R}^{+-, n_{2} n_{2}} T_{R L}^{a, n_{2} n}$. We arbitrarily chose the island charge indices on $\sigma_{I R}$ as $n+1$ and $n$, which makes it an electron transfer, and consequently the complete product must be of $e e$-type. Fixing $\sigma$ as $e$-type, the inner charge index could as well be taken as being summed over because an electron transfer only allows I to appear with charge index $n+1: G_{R I}^{+-, n n+1} \sigma_{I R}^{n+1 n}=\left(G_{R I}^{+-, n n_{2}} \sigma_{I R}^{n_{2}{ }^{n}}\right)_{e e}$. The four products in the text above have been noted with the respective outer island charge index $n$ or $n+1$. We understand that finally the $e e$-part is taken, although for inner $e h$-indices all possible combinations get summed over. We give the treatment of the first term as an example. Rate terms require $G^{+-}\left(\tau, \tau^{\prime}\right) \sigma\left(\tau^{\prime}\right)$ of two identical time arguments, that is $\tau=\tau^{\prime}$ [12]. Charging rates were expressed as expectation values (equation (12)), which are, of course, taken for the state of the system at time $\tau$. Working in the interaction picture here, $G^{+-}$refers that state back to infinitely long ago when the coupling between L, I and R was turned on. $G^{+-}$(with one index $\mathrm{L}$ or $\mathrm{R}$ and $\mathrm{I}$ the other) describe all the rest of the circuit on the Keldysh contour except the rightmost $\sigma$ on the time axis (see figure 2). $G^{+-}$is of $(\tau, \tau)$ to frame $\sigma(\tau)$. The definition (7) of $G^{+-}$was usefully made for general $\left(\tau, \tau^{\prime}\right)$ as all other Green's functions $G^{r / a}$ and $g^{r / a /+-}$ are of two time arguments, too. The first of the four rate contributions becomes:

$$
\begin{aligned}
\sum_{n_{2}} \int \mathrm{d} \tau_{1} \mathrm{~d} \tau_{2} \mathrm{~d} \tau_{3} g_{R R}^{r, n n}\left(\tau, \tau_{1}\right) T_{R L}^{r, n n_{2}}\left(\tau_{1}, \tau_{2}\right) g_{L L}^{+-, n_{2} n_{2}}\left(\tau_{2}, \tau_{3}\right) T_{L R}^{a, n_{2} n}\left(\tau_{3}, \tau\right) \\
=\sum_{n_{2}} \int \mathrm{d} \tau_{1} \mathrm{~d} \tau_{2} \mathrm{~d} \tau_{3} \mathrm{~d} \omega \mathrm{d} \omega_{1} \mathrm{~d} \omega_{2} \mathrm{~d} \omega_{3} \sum_{\substack{k, p \\
k+p=n_{2}-n}} \sum_{\substack{k^{\prime}, p^{\prime} \\
k^{\prime}+p^{\prime}=n-n_{2}}} \mathrm{e}^{-\mathrm{i} \omega \tau} \mathrm{e}^{\mathrm{i} \omega \tau_{1}} g_{R R}^{r, n n}(\omega) \\
\cdot \mathrm{e}^{-\mathrm{i} \omega_{1} \tau_{1}} \mathrm{e}^{\mathrm{i} k A \tau_{2}} \mathrm{e}^{\mathrm{i} m B \tau_{2}} \mathrm{e}^{\mathrm{i} p C \tau_{2}} \mathrm{e}^{\mathrm{i} \omega_{1} \tau_{2}}\left(T_{L R, k p}^{a, n_{2} n}\left(\omega_{1}\right)\right)^{\dagger} \mathrm{e}^{-\mathrm{i} \omega_{2} \tau_{2}} \mathrm{e}^{\mathrm{i} \omega_{2} \tau_{3}} g_{L L}^{+-, n_{2} n_{2}}\left(\omega_{2}\right) \\
\cdot \mathrm{e}^{-\mathrm{i} \omega_{3} \tau_{3}} \mathrm{e}^{-\mathrm{i} k^{\prime} A \tau_{3}} \mathrm{e}^{-\mathrm{i} m^{\prime} B \tau_{3}} \mathrm{e}^{-\mathrm{i} p^{\prime} C \tau_{3}} \mathrm{e}^{\mathrm{i} \omega_{3} \tau} T_{L R, k p}^{a, n_{2} n}\left(\omega_{3}\right) .
\end{aligned}
$$


Recognizing transcriptions of $\delta$-distributions eliminates all but the $\omega$-integral and requires $\omega_{1}=\omega, \omega_{2}=\omega+k A+m B+p C, \omega_{3}=\omega+\left(k-k^{\prime}\right) A+\left(m-m^{\prime}\right) B+\left(p-p^{\prime}\right) C$. The remaining phase factor is $\mathrm{e}^{-\mathrm{i} \omega \tau} \mathrm{e}^{\mathrm{i} \omega_{3} \tau}$. Only the dc part counts for island charging, which further imposes $\omega_{3}=\omega$. As $A, B, C$ are assumed incommensurate ${ }^{3}$, this implies $k^{\prime}=k$ and $p^{\prime}=p$, which in turn automatically ensures $m^{\prime}=m$. Summing over $k$ and $p$ independently without restriction is equivalent to summing over $n_{2}$ (see (17) for the result). The purpose of the careful choice of Fourier representations for the $T$ was in all terms to make the remaining frequency $\omega$ to be integrated over the argument of both $T$-factors as well as the argument of $g_{R R}$, be it the $r$ - or the +- -function. The two $T$-factors in each product actually become one and the same function, but for our special $\dagger$-transposition. This property is convenient to keep the effort to calculate $T$-functions [11] as low as possible. Having $g_{R R}(\omega)$ in all of them is to ensure consistency between the four terms from (10) and (11), respectively, listed in the text above. With that recipe identical integration boundaries on all four terms-an actual calculation requires finite limits - guarantee that contributions tending towards non-zero constant values for $\omega \rightarrow \pm \infty$ cancel on the correct half-open intervals (see the next section). Adding the hole contribution, the complete rate to change the island charge between $n$ and $n+1$ by a transfer through the right junction is

$$
\begin{aligned}
2 \operatorname{Re}\left\{\left(\sigma_{R I} G_{I R}^{+-}\right.\right. & )_{h h}^{n+1}+\left(G_{R I}^{+-} \sigma_{I R}\right)_{e e}^{n}\right)\right\} \\
= & 2 \operatorname{Re}\left(T_{R I}^{r} g_{I I}^{+-} T_{I R}^{a} g_{R R}^{a}\right)_{h h}^{n+1}+\left(g_{I I}^{r} T_{I R}^{r} g_{R R}^{+-} T_{R I}^{a}\right)_{h h}^{n}+\left(T_{R L}^{r} g_{L L}^{+-} T_{L R}^{a} g_{R R}^{a}\right)_{h h}^{n+1} \\
& -\left(T_{L R}^{r} g_{R R}^{+-} T_{R L}^{a} g_{L L}^{a}\right)_{h h}^{n+1}+\left(g_{R R}^{r} T_{R I}^{r} g_{I I}^{+-} T_{I R}^{a}\right)_{e e}^{n}+\left(T_{I R}^{r} g_{R R}^{+-} T_{R I}^{a} g_{I I}^{a}\right)_{e e}^{n+1} \\
& +\left(g_{R R}^{r} T_{R L}^{r} g_{L L}^{+-} T_{L R}^{a}\right)_{e e}^{n}-\left(g_{L L}^{r} T_{L R}^{r} g_{R R}^{+-} T_{R L}^{a}\right)_{e e}^{n}=2 \operatorname{Re} \int \mathrm{d} \omega \sum_{p, k}\{ \\
& {\left[\left(T_{a, I R, k p}^{n+1-k-p n+1}\right)^{\dagger} g_{I I}^{+-}\left(\omega^{\prime}\right) T_{a, I R, k p}^{n+1-k-p n+1} g_{R R}^{a}\right]_{h h} \quad m=-(k+p)(n+1-k-p)-(k+p)^{2} / 2+1 / 2 } \\
& +\left[g_{I I}^{r}\left(\omega^{\prime}\right) T_{r, I R, k p}^{n+k+k} g_{R R}^{+-}\left(T_{r, I R, k p}^{n n+k+p}\right)^{\dagger}\right]_{h h} \quad m=-(k+p) n-(k+p)^{2} / 2+1 / 2 \\
& +\left[\left(T_{a, L R, k p}^{n+1-k-p n+1}\right)^{\dagger} g_{L L}^{+-}\left(\omega^{\prime}\right) T_{a, L R, k p}^{n+1-k-p} n+1 g_{R R}^{a}\right]_{h h} \quad m=-(k+p)(n+1-k-p)-(k+p)^{2} / 2 \\
& -\left[T_{r, L R, k p}^{n+1 n+1+k+p} g_{R R}^{+-}\left(T_{r, L R, k p}^{n+1 n+1+k+p}\right)^{\dagger} g_{L L}^{a}\left(\omega^{\prime}\right)\right]_{h h} \quad m=-(k+p)(n+1)-(k+p)^{2} / 2 \\
& +\left[g_{R R}^{r}\left(T_{a, I R, k p}^{n-k-p}\right)^{\dagger} g_{I I}^{+-}\left(\omega^{\prime}\right) T_{a, I R, k p}^{n-k-p}\right]_{e e} \quad m=-(k+p)(n-k-p)-(k+p)^{2} / 2+1 / 2 \\
& +\left[T_{r, I R, k p}^{n+1 n+1+k+p} g_{R R}^{+-}\left(T_{r, I R, k p}^{n+1 n+1+k+p}\right)^{\dagger} g_{I I}^{a}\left(\omega^{\prime}\right)\right]_{e e} \quad m=-(k+p)(n+1)-(k+p)^{2} / 2+1 / 2 \\
& +\left[g_{R R}^{r}\left(T_{a, L R, k p}^{n-k-p n}\right)^{\dagger} g_{L L}^{+-}\left(\omega^{\prime}\right) T_{a, L R, k p}^{n-k-p}\right]_{e e} \quad m=-(k+p)(n-k-p)-(k+p)^{2} / 2 \\
& \left.-\left[g_{L L}^{r}\left(\omega^{\prime}\right) T_{r, L R, k p}^{n n+k+p} g_{R R}^{+-}\left(T_{r, L R, k p}^{n n+k+p}\right)^{\dagger}\right]_{e e} \quad m=-(k+p) n-(k+p)^{2} / 2\right\}
\end{aligned}
$$

$m$-values are indicated with each term (with $k$ even and $p$ odd on $T_{I R}$ and $k$ and $p$ both odd on $T_{L R}, m$ is always integer). $\omega^{\prime}$ means $\omega+k A+m B+p C$ in each case and left out arguments of $g$ - and $T$-functions are $\omega$. The same formula with indices $\mathrm{L}$ and $\mathrm{R}$ as well as voltage drops $A$ and $C$ interchanged, holds for charging rates through the left junction. For the normal

3 Even if there might be resonances when Fermi levels of a lead and an island charge state match, these would have to have some finite width. In that sense transfer functions, charging rates and currents should be continuous functions of the voltages between each lead and the island, and also of these in relation to the charging energy. There is no restriction in excluding multiples of one another amongst $A, B$ and $C$, because such a very special situation is already destroyed by any infinitesimally small parameter change. Singularity matching is included as the limiting case of just incommensurate voltages. (Eventually later including $\delta$-peak like DOS-contributions for Cooper pairs may again require some care at this point.) 
state the complete expression (17) with $e e$ - and $h h$-part-in the incoherent model [10] the respective formula without the $T_{L R}$-terms - in the limit $\Delta \rightarrow 0$ must be used. Comparison to the single-electron transistor in the low transmission limit reveals that the ee-part alone would not yield the energetically correct charging thresholds. (With the island potential changing with charging even for $\Delta=0$ the $h h$-part does not simply double the ee-part.)

The form of the rates (17) suggests the following interpretation: coherence shows itself in the fact that the right lead effectively couples [15] to the left lead as it does to the island, although to the former it is only indirectly connected. Terms $\left(g_{R R}^{r} T_{R I}^{r} g_{I I}^{+-} T_{I R}^{a}\right)_{e e}^{n}$ and $\left(g_{R R}^{r} T_{R L}^{r} g_{L L}^{+-} T_{L R}^{a}\right)_{e e}^{n}$ as well as $\left(T_{R I}^{r} g_{I I}^{+-} T_{I R}^{a} g_{R R}^{a}\right)_{h h}^{n+1}$ and $\left(T_{R L}^{r} g_{L L}^{+-} T_{L R}^{a} g_{R R}^{a}\right)_{h h}^{n+1}$, respectively, look the same but for I replaced by $L$. Charge carriers having originated [12] in L and I can on their course both be those being transported across the right junction. Terms $\left(T_{I R}^{r} g_{R R}^{+-} T_{R I}^{a} g_{I I}^{a}\right)_{e e}^{n+1}$ and $\left(g_{I I}^{r} T_{I R}^{r} g_{R R}^{+-} T_{R I}^{a}\right)_{h h}^{n}$ count hoppings of carriers stemming from the right lead, however through both junctions. For the rate to charge the island through the right junction transfers of those carriers across the left, $\left(g_{L L}^{r} T_{L R}^{r} g_{R R}^{+-} T_{R L}^{a}\right)_{e e}^{n}$ and $\left(T_{L R}^{r} g_{R R}^{+-} T_{R L}^{a} g_{L L}^{a}\right)_{h h}^{n+1}$, have to be subtracted (see also figure 2).

\section{Interference correction and crossed Andreev reflection}

\subsection{Multiple electron and hole reflection}

It is the virtue of the Green's functions method that interaction terms do not get cut at a finite order like in a perturbation approach. However, not for numerical calculations, but for an intuitive interpretation of the results from our model [14], for enlightening the chimerical issue of choosing frequency arguments and integration boundaries consistently, and also for taking up the cudgels for the implementation of the rate formula (17), an analytic development of some low-order contributions proves indispensable. Lowest-order terms with just replacing each $T$ in (17) by a single $\sigma$ can only stem from $\left(T_{R I}^{r} g_{I I}^{+-} T_{I R}^{a} g_{R R}^{a}\right)_{h h}^{n+1},\left(g_{I I}^{r} T_{I R}^{r} g_{R R}^{+-} T_{R I}^{a}\right)_{h h}^{n},\left(g_{R R}^{r} T_{R I}^{r} g_{I I}^{+-} T_{I R}^{a}\right)_{e e}^{n}$ and $\left(T_{I R}^{r} g_{R R}^{+-} T_{R I}^{a} g_{I I}^{a}\right)_{e e}^{n+1}$. Lowest orders in $T_{L R}$ and $T_{R L}$ are $\sigma_{L I} g_{I I} \sigma_{I R}$ and $\sigma_{R I} g_{I I} \sigma_{I L}$, respectively, and thus the lowest-order contributions in (17) from the terms with $T_{L R / R L}$ contain $4 \sigma$, while the leading ones out of terms with $T_{I R / R I}$ are products with $2 \sigma$. The $2 \sigma$-parts are exactly the same as in the incoherent model [10] and the condition that such transfers across the right junction being single hoppings in themselves - holes and electrons taken together-change the island charge from $n$ to $n+1$ is $2 C>4 \Delta+4 n E_{C}$ and $2 C>(4 n+2) E_{C}$. For decharging from $n+1$ to $n$ conditions are $2 C<4(n+1) E_{C}-4 \Delta$ and $2 C<(4 n+2) E_{C}$. Voltages $A, B$ and $C$ are understood to be multiplied by $e$ when compared to energies $E_{C}$ and $\Delta$.

$4 \sigma$-terms out of $\left(T_{I R}^{r} g_{R R}^{+-} T_{R I}^{a} g_{I I}^{a}\right)_{e e}^{n+1}$ and $\left(g_{R R}^{r} T_{R I}^{r} g_{I I}^{+-} T_{I R}^{a}\right)_{e e}^{n}$ mean developing one $T$ into a product $\sigma g \sigma g \sigma$ and replacing the other by a single $\sigma$. For brevity, only the electron terms from (17) will be written out. The reader can easily infer the treatment of the hole terms. We shall first look at $4 \sigma$ terms only involving the I- and R-index and without AR:

(1) $g_{R R}^{r, e e} \sigma_{R I, e}^{n n+1} g_{I I}^{r, e e} \sigma_{I R, e}^{n+1 n} g_{R R}^{r, e e} \sigma_{R I, e}^{n n+1} g_{I I}^{+-, e e} \sigma_{I R, e}^{n+1 n} \rightarrow g_{R R}^{r, e e}() g_{I I}^{r, e e}() g_{R R}^{r, e e}() g_{I I}^{+-, e e}()$

(2) $g_{R R}^{r, e e} \sigma_{R I, e}^{n n+1} g_{I I}^{+-, e e} \sigma_{I R, e}^{n+1 n} g_{R R}^{a, e e} \sigma_{R I, e}^{n n+1} g_{I I}^{a, e e} \sigma_{I R, e}^{n+1 n} \rightarrow g_{R R}^{r, e e}() g_{I I}^{+-, e e}() g_{R R}^{a, e e}() g_{I I}^{a, e e}()$

(3) $\quad \sigma_{I R}^{n+1 n} g_{R R}^{r, e e} \sigma_{R I, e}^{n n+1} g_{I I}^{r, e e} \sigma_{I R, e}^{n+1 n} g_{R R}^{+-, e e} \sigma_{R I, e}^{n n+1} g_{I I}^{a, e e} \rightarrow g_{R R}^{r, e e}() g_{I I}^{r, e e}() g_{R R}^{+-, e e}() g_{I I}^{a, e e}()$

(4) $\sigma_{I R}^{n+1 n} g_{R R}^{+-, e e} \sigma_{R I, e}^{n n+1} g_{I I}^{a, e e} \sigma_{I R, e}^{n+1 n} g_{R R}^{a, e e} \sigma_{R I, e}^{n n+1} g_{I I}^{a, e e} \rightarrow g_{R R}^{+-, e e}() g_{I I}^{a, e e}() g_{R R}^{a, e e}() g_{I I}^{a, e e}()$.

Here is the recipe to obtain the frequency arguments of the two $g$ not occurring in (17): understanding that all $\omega$ and $\tau$ (except $\tau$ without index) are integrated over, writing $\sigma$ explicitly and replacing the $g$ by their Fourier representations with initially unknown frequency 


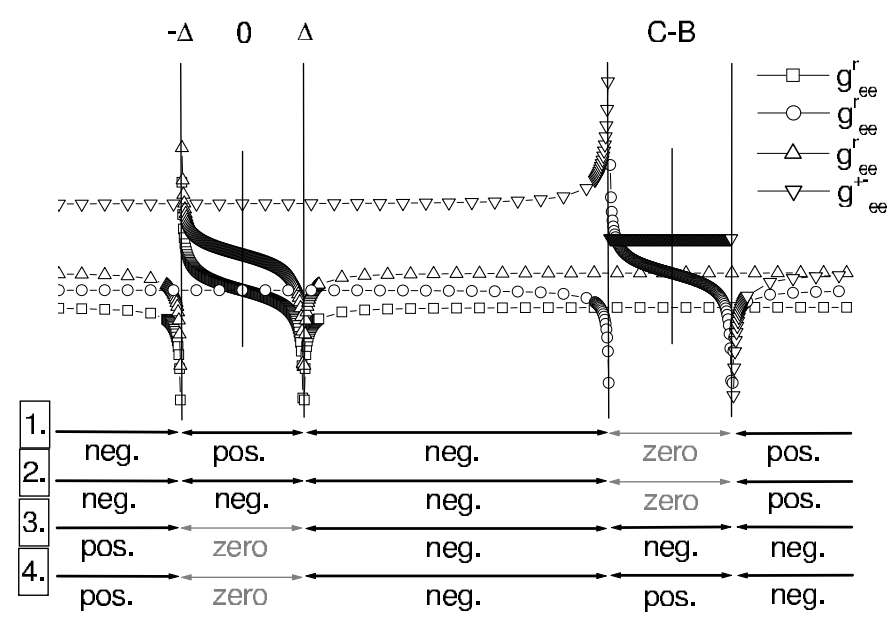

Figure 3. Products from terms (18) with $n=0$. The functions are shifted vertically. Refer to figure 9 (appendix) for zero levels and for distinguishing $\operatorname{Re} g$ and $\operatorname{Im} g$.

arguments except for the $g_{R R}(\omega)$ from (17), the first line of (18), for example, translates into: $\begin{aligned} \mathrm{e}^{-\mathrm{i} \omega \tau} \mathrm{e}^{\mathrm{i} \omega \tau_{1}} g_{R R}^{r, e e}(\omega) t_{R} \mathrm{e}^{-\mathrm{i} C \tau_{1}} \mathrm{e}^{\mathrm{i}(n+1) B \tau_{1}} \mathrm{e}^{-\mathrm{i} \omega_{1} \tau_{1}} \mathrm{e}^{\mathrm{i} \omega_{1} \tau_{2}} g_{I I}^{r, e e}\left(\omega_{1}\right) t_{R} \mathrm{e}^{\mathrm{i} C \tau_{2}} \mathrm{e}^{-\mathrm{i}(n+1) B \tau_{2}} & \\ & \times \mathrm{e}^{-\mathrm{i} \omega_{2} \tau_{2}} \mathrm{e}^{\mathrm{i} \omega_{2} \tau_{3}} g_{R R}^{r, e e}\left(\omega_{2}\right) t_{R} \mathrm{e}^{-\mathrm{i} C \tau_{3}} \mathrm{e}^{\mathrm{i}(n+1) B \tau_{3}} \mathrm{e}^{-\mathrm{i} \omega_{3} \tau_{3}} \mathrm{e}^{\mathrm{i} \omega_{3} \tau} g_{I I}^{+-, e e}\left(\omega_{3}\right) t_{R} \mathrm{e}^{\mathrm{i} C \tau} \mathrm{e}^{-\mathrm{i}(n+1) B \tau} .\end{aligned}$

Recognizing $\delta$-distributions, which leave only the $\omega$-integration, then enforces $\omega_{1}=$ $\omega-C+(n+1) B, \omega_{2}=\omega_{1}+C-(n+1) B=\omega$ and $\omega_{3}=\omega_{(2)}-C+(n+1) B$. The remaining phase exponentials in (19) then cancel. On the right-hand side of (18) we omitted the factor $t_{R}^{4}$ in all lines. The voids for the arguments have to be filled with $\omega$ for all $g_{R R}$ and $\omega-C+(n+1) B$ for all $g_{I I}$. Now we draw the $g$-functions appearing on the right-hand side of (18) (figure 3 ). Under the plot we note for each interval where the product is real, whether it is positive or negative. The plot is only shown for the first of the four terms from (18), the sign of the products is noted for all of them. Even if a term in an interval has opposite sign from term No. 1, due to the unique shape of the $g$-functions, each term at a fixed frequency $\omega$ produces the same absolute value. Most important is the fact that in the $\omega$-integration the parts from the outermost half-open intervals cancel. This is essential for contributions without AR as the $g$-products tend to finite values for $\omega \rightarrow \pm \infty$. The products can have real non-vanishing values inside a $2 \Delta$-interval where two $g$-functions are centered; however, contributions cancel each other here. Drawn for the case that $C-2 E_{C}>0$ and for non-overlapping $2 \Delta$-intervals the middle interval produces a negative rate. Figure 3 is drawn for a different case from the one we usually regard, namely the applied voltage poled the other way such that, here compared to figure 5, the Fermi level of the right lead lies above that of the involved island state. In that situation the island should be charged up by electrons coming from the right and holes going to the right. The respective aforementioned $2 \sigma$ rate contribution is indeed positive. Here we see the next-order correction to that, which has opposite sign (see the third term in (22) as compared to the first). (Rate contributions are not and must not be separated into different orders of processes when entered into the rate matrix.) The $2 \sigma$-terms have their $g$-functions centered at the same frequencies as the kind of $4 \sigma$-terms from (18) and figure 3 [10]. The electron contribution has the non-zero center frequency at $C-(n+1) B$, whereas the hole part has it at $-C+n B$. Of the given conditions for single particle hopping $2 C>4 \Delta+4 n E_{C}$ 
or $2 C<4(n+1) E_{C}-4 \Delta$ refer to the middle interval between the two $\Delta$-gaps appearing at certain voltages in such ways that I is charged up by hole transfers and decharged by electron transfers, respectively. $-C+n B$ and $C-(n+1) B$ can fall on the same side of zero. Then, if existing, middle intervals give contributions of opposite sign for electrons and holes. At $2 C=(4 n+2) E_{C}$ these middle intervals become of equal length and then their contributions cancel. Expressed in a level diagram this means that the single-particle charging rate from $n$ to $n+1$ across a junction changes sign when that lead's Fermi level is at the height midway between the island's $n$ and $n+1$ levels. These explanations were just given to review the illustration of rate contributions as $g$-function plots and their connection to energy ranges in the level diagram using the most simple example.

All the more interesting are the $4 \sigma$-contributions containing $g_{R R}$ and $g_{L L}$ at the same time. We first look at those without AR and in an exemplary way this time take those from the hole parts of (17). From $\left(T_{R L}^{r} g_{L L}^{+-} T_{L R}^{a} g_{R R}^{a}\right)_{h h}^{n+1}$ and $-\left(T_{L R}^{r} g_{R R}^{+-} T_{R L}^{a} g_{L L}^{a}\right)_{h h}^{n+1}$ there are:

(1) $\sigma_{R I, h}^{n+1 n} g_{I I}^{r, h h} \sigma_{I L, h}^{n n+1} g_{L L}^{+-, h h} \sigma_{L I, h}^{n+1 n} g_{I I}^{a, h h} \sigma_{I R, h}^{n n+1} g_{R R}^{a, h h} \rightarrow g_{I I}^{r, h h}() g_{L L}^{+-, h h}() g_{I I}^{a, h h}() g_{R R}^{a, h h}()$

(2) $\quad-\sigma_{L I, h}^{n+1 n} g_{I I}^{r, h h} \sigma_{I R, h}^{n n+1} g_{R R}^{+-, h h} \sigma_{R I, h}^{n+1 n} g_{I I}^{a, h h} \sigma_{I L, h}^{n n+1} g_{L L}^{a, h h} \rightarrow-g_{I I}^{r, h h}() g_{R R}^{+-, h h}() g_{I I}^{a, h h}() g_{L L}^{a, h h}()$.

We recall that in the coherent model $T_{R I / I R}$ contain backreflections to the left lead and thus $\left(g_{I I}^{r} T_{I R}^{r} g_{R R}^{+-} T_{R I}^{a}\right)_{h h}^{n}$ and $\left(T_{R I}^{r} g_{I I}^{+-} T_{I R}^{a} g_{R R}^{a}\right)_{h h}^{n+1}$ further contribute:

(3) $\quad g_{I I}^{r, h h} \sigma_{I L, h}^{n n+1} g_{L L}^{r, h h} \sigma_{L I, h}^{n+1 n} g_{I I}^{r, h h} \sigma_{I R, h}^{n n+1} g_{R R}^{+-, h h} \sigma_{R I, h}^{n+1 n} \rightarrow g_{I I}^{r, h h}() g_{L L}^{r, h h}() g_{I I}^{r, h h}() g_{R R}^{+-, h h}()$

(4) $g_{I I}^{r, h h} \sigma_{I R, h}^{n n+1} g_{R R}^{+-, h h} \sigma_{R I, h}^{n+1 n} g_{I I}^{a, h h} \sigma_{I L, h}^{n n+1} g_{L L}^{a, h h} \sigma_{L I, h}^{n+1 n} \rightarrow g_{I I}^{r, h h}() g_{R R}^{+-, h h}() g_{I I}^{a, h h}() g_{L L}^{a, h h}()$

(5) $\sigma_{R I, h}^{n+1 n} g_{I I}^{r, h h} \sigma_{I L, h}^{n n+1} g_{L L}^{r, h h} \sigma_{L I, h}^{n+1 n} g_{I I}^{+-, h h} \sigma_{I R, h}^{n n+1} g_{R R}^{a, h h} \rightarrow g_{I I}^{r, h h}() g_{L L}^{r, h h}() g_{I I}^{+-, h h}() g_{R R}^{a, h h}()$

(6) $\quad \sigma_{R I, h}^{n+1 n} g_{I I}^{+-, h h} \sigma_{I L, h}^{n n+1} g_{L L}^{a, h h} \sigma_{L I, h}^{n+1 n} g_{I I}^{a, h h} \sigma_{I R, h}^{n n+1} g_{R R}^{a, h h} \rightarrow g_{I I}^{+-, h h}() g_{L L}^{a, h h}() g_{I I}^{a, h h}() g_{R R}^{a, h h}()$.

Having fixed the argument of the $g_{R R}$ from (17) to $\omega$ in all six terms, the argument of all $g_{R R}$ is $\omega$, all $g_{L L}$ are taken of $\omega-A+C$ and all $g_{I I}$ of $\omega+C-n B$. A factor $t_{L}^{2} t_{R}^{2}$ for two hoppings across each of the junctions was omitted in noting the terms on the right. Contributions 2 and 4 cancel, the other four are sketched in figure 4 for $n=0$. For illustration we chose the easiest situation where the $2 \Delta$-intervals around $0,-C$ and $A-C$ are on the $\omega$-axis in that order and do not overlap. (For the case that the neutral island state $n=0$ is situated between the left and the right lead in electrostatic potential, in our model $A$ is a positive quantity and $C$ a negative one. $n=0$ is the island state involved in the example here.) In figure 4 the middle $2 \Delta$ - and the two neighboring intervals give non-vanishing contributions. The net value from the left interval has opposite sign from the other intervals. For interpretation contributions are better taken as a negative one over the whole range canceled by a twice as large positive one in the left interval. Figure 5 displays these in the energy-level diagram. In the entire range between the superconductor gaps of the leads holes can go from filled states right to empty ones left. The first half across the right junction of this transition intermittently changes the island charge by one unit, even if the hole does not find a state to relax into on the island. The charge carrier can travel through the island at energies inside or outside the gap. The only condition here is that the lead gaps do not overlap, that is a voltage greater than $2 \Delta / e$. Despite the applied voltage bridging two superconductor junctions, only once $2 \Delta / e$ is needed. If there is an energy range between the gaps of the island state involved and the right lead, there are other transport processes in this interval the interference of which is of the same order. (For an intensity of the hole transfer from lead to lead the product of both junction transmissions has to be squared.) Between the right lead and the island, single hole transfer can interfere with a hole transfer extended by a forth- and backhopping to the left lead, nevertheless ending on the island. Hole transfers onto the island decrease the island charge (counted in electrons) and 


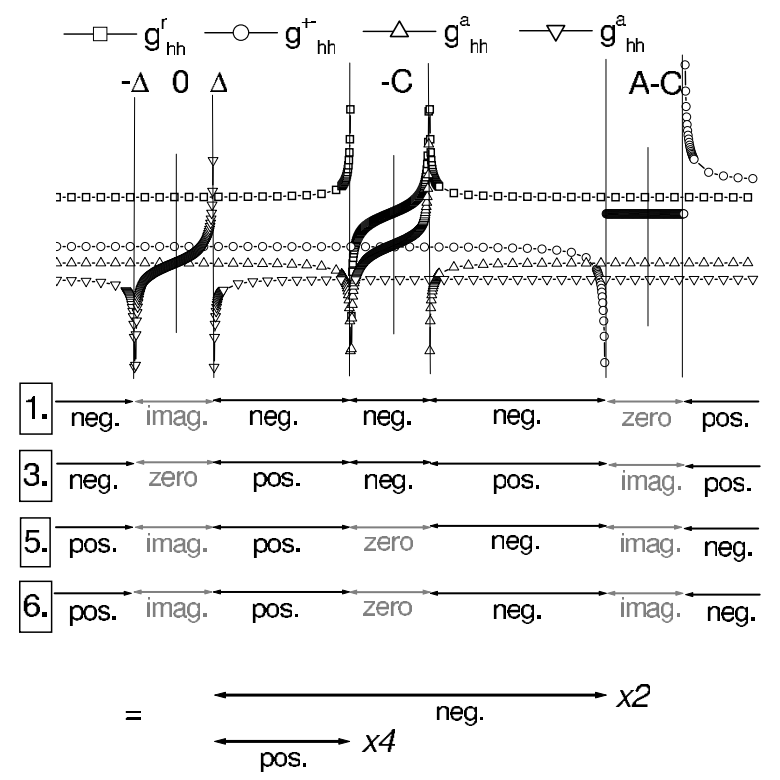

Figure 4. Products from terms (20) for $n=0$.

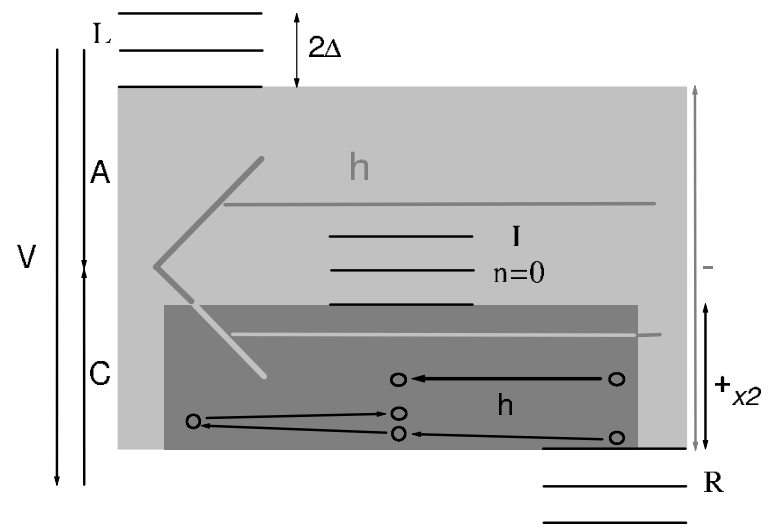

Figure 5. Transport processes behind terms (20).

therefore their contributions to $n \rightarrow n+1$ charging rates come out negative. The interference with the process with backreflection from the left lead we find to be destructive to single hole transfer across the right junction as well as from lead to lead. Its contribution to the charging rate has opposite sign. Having nothing to do with AR, this phenomenon is equally present in the normal state or $\Delta \rightarrow 0$ limit of our model. In this case it is possible to calculate transfer functions [11] analytically. They turn out to be energy independent, and from their Dyson equation in frequency space one gets (separation into $e$ and $h$ is not necessary here)

$T_{L I / I L}^{r / a, N}=\frac{t_{L}}{1+t_{L}^{2}+t_{R}^{2}}, \quad T_{R I / I R}^{r / a, N}=\frac{t_{R}}{1+t_{L}^{2}+t_{R}^{2}}, \quad T_{L R / L R}^{r / a, N}=\frac{t_{L} t_{R}}{1+t_{L}^{2}+t_{R}^{2}}$

Factors $i$ need not be cared about, because they get canceled by complex conjugates in rate terms, anyway. (The first two quantities should be compared to the stand-alone junctions or 
the incoherent case where we would have the greater values $T_{L I / I L}=\frac{t_{L}}{1+t_{L}^{2}}$ and $T_{R I / I R}=\frac{t_{R}}{1+t_{R}^{2}}$.) The intensity for transfers between the right lead and the island in an energy interval with respective filled and empty states is thus proportional to

$$
\left(\frac{t_{R}}{1+t_{L}^{2}+t_{R}^{2}}\right)^{2}=t_{R}^{2}-\mathbf{2} \mathbf{t}_{\mathbf{L}}^{2} \mathbf{t}_{\mathbf{R}}^{2}-2 t_{R}^{4} \pm \cdots
$$

The intensity for coherent transfers between the right and left leads is determined by

$$
\left(\frac{t_{L} t_{R}}{1+t_{L}^{2}+t_{R}^{2}}\right)^{2}=\mathbf{t}_{\mathbf{L}}^{2} \mathbf{t}_{\mathbf{R}}^{2}-2 t_{L}^{2} t_{R}^{4}-2 t_{L}^{4} t_{R}^{2} \pm \cdots
$$

Even though each junction transmission, that is $T_{L I / I L}$ or $T_{R I / I R}$, is already renormalized in a way to also take into account multiple reflections across the other junction, coherence over the whole system further means - besides transport across each junction-the presence of direct transport from lead to lead. The latter then gets broken up into two parts when considering the electrostatical island charging, though. The addition of contributions of both kinds as described by (22) and (23) applies to the superconducting state, too, as already discussed with rate terms (17). The dark grey range in figure 4 corresponds to the bold-faced term in (22), the light grey range to the first term in (23). The developments confirm the relative sign of the terms. The analogous electron transfer processes to (20) can be illustrated in an image like figure 5 involving the $n+1$ island state. In the normal state, where $T$-functions and rate terms can be calculated in closed forms, for the special case $\theta_{1}=\theta_{2}=1$ or $t_{L}=t_{R}=1$ (see [12, 14] for conversion between $t$ and $\theta$ ) (22) and (23) give equal values. Therefore the size of the energy intervals where transport from filled to empty states is possible alone decides about the direction of net charge flows. We now consider the symmetric case with the neutral island's Fermi level midway between those of the leads and such a low voltage that the middle $(0 \leftrightarrow 1)$ between the $n=0$ and $n=1$ states is still above the left Fermi level. Electron-like charges from below that middle level and above the left level flow from I to L. Taking that middle level comprises the effects from electron and hole terms for $0 \leftrightarrow 1$ charge changes of I into a single picture [10]. Charges between the L and R Fermi levels can travel from $\mathrm{L}$ to $\mathrm{R}$, intermittently charging $\mathrm{I}$ from 0 to 1 . In electron units holes going from $\mathrm{R}$ to $\mathrm{L}$ are counted as negative charges going from $\mathrm{L}$ to $\mathrm{R}$ the same as electrons. The condition that the interval between the $\mathrm{L}$ and $\mathrm{R}$ Fermi levels be larger than the one above it with flow in opposite direction reads $e V>E_{C}-\frac{1}{2} e V$ or $e V>\frac{2}{3} E_{C}$ (figure 6). In contrast to figures 3-5 and the related discussions we here compare complete rate terms including processes of all order. In the normal state these are easily evaluated with the analytically known $T$-functions; without $e h$-conversion from AR only $k$ and $p$ equal to $0, \pm 1$ appear. Decharging to the right is obviously no problem with $\mathrm{R}$ below all other levels in figure 6 . This proves that in our model the earliest possible current onset is at $e V=\frac{2}{3} E_{C}$. A Coulomb blockade effect is still felt despite coherent transport from lead to lead contributing. For other cases than $\theta_{1}=\theta_{2}=1$ weight factors enter the counterbalancing of energy interval lengths and the current onset threshold depends on the transmissions. In the incoherent model, in normal state identical to orthodox theory, no current can flow up to $e V=2 E_{C}$, which is the well-known Coulomb blockade phenomenon. From figure 5 we learn that it depends on the position of the involved island charge state whether direct lead-to-lead transport can enhance a charging rate-and thus eventually the current_as compared to the setup with merely incoherently coupled junctions. An easy rule when the intervals from figure 4, that is terms (20), together with the corresponding electron terms will give a positive or negative rate contribution cannot be given. A part in the current which increases linearly with voltage already in the subgap range and over a scale much larger than $E_{C}$ is indeed observed if the charging energy is rather 


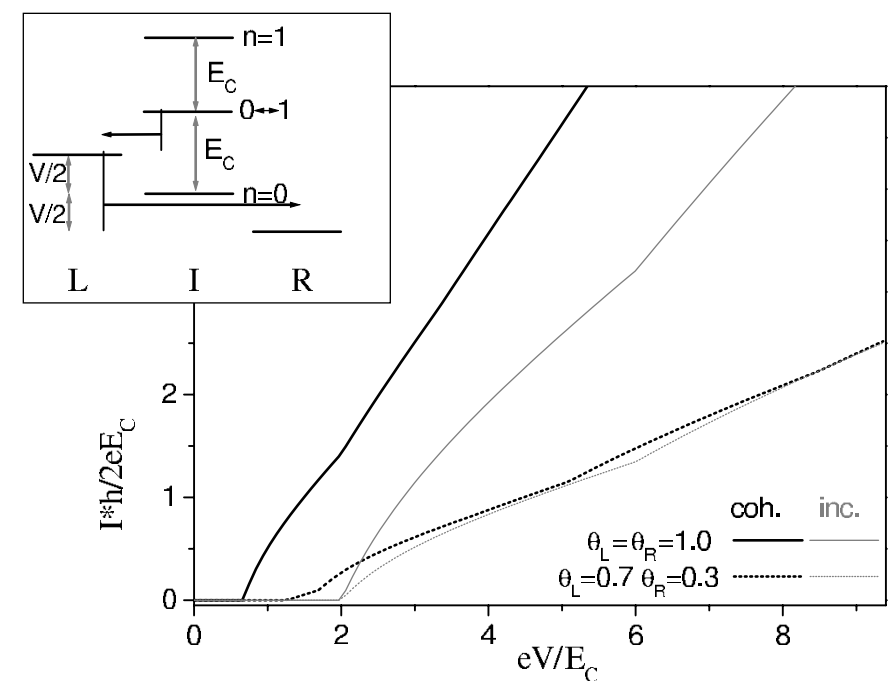

Figure 6. Calculated $I V$-characteristics in normal state for fully open channels in both junctions as well as an example with different transmissions. Results from the incoherent model [10] added for comparison. Inset: for the neutral island in the middle between the lead Fermi levels (equal junction capacitances, no gate, as for all curves here) and transmissions $\theta_{1}=\theta_{2}=1$ intervals with charge flow in opposite directions are compared in order to get the voltage threshold for charging I from 0 to 1 across the left junction to become possible.

small compared to the gap, see the examples in [14], and is associated with direct lead-to-lead transport.

\subsection{Lowest-order Andreev reflection}

$4 \sigma$-terms with AR only between the island and one lead have been presented in [10]. Here we shall analyze the lowest-order AR terms inherent to the coherent model, that is those involving all three sites. Again, only those from (17) counted as an electron transfer, that is those with outer index $e$, are written in detail in an exemplary way.
from $\left(g_{R R}^{r} T_{R L}^{r} g_{L L}^{+-} T_{L R}^{a}\right)_{e e}^{n}$ :
1. $g_{R R}^{r, e e} \sigma_{R I, e}^{n n+1} g_{I I}^{r, e h} \sigma_{I L, h}^{n+1 n+2} g_{L L}^{+-, h h} \sigma_{L I, h}^{n+2 n+1} g_{I I}^{a, h e} \sigma_{I R, e}^{n+1 n}$
from $-\left(g_{L L}^{r} T_{L R}^{r} g_{R R}^{+-} T_{R L}^{a}\right)_{e e}^{n}:$
2. $-g_{L L}^{r, e e} \sigma_{L I, e}^{n n+1} g_{I I}^{r, e h} \sigma_{I R, h}^{n+1 n+2} g_{R R}^{+-, h h} \sigma_{R I, h}^{n+2 n+1} g_{I I}^{a, h e} \sigma_{I L, e}^{n+1 n}$
from $\left(T_{I R}^{r} g_{R R}^{+-} T_{R I}^{a} g_{I I}^{a}\right)_{e e}^{n+1}$ :
3. $\sigma_{I L, e}^{n+1 n} g_{L L}^{r, e e} \sigma_{L I, e}^{n n+1} g_{I I}^{r, e h} \sigma_{I R, h}^{n+1 n+2} g_{R R}^{+-, h h} \sigma_{R I, h}^{n+2 n+1} g_{I I}^{a, h e}$
4. $\sigma_{I R, e}^{n+1 n} g_{R R}^{+-, e e} \sigma_{R I, e}^{n n+1} g_{I I}^{a, e h} \sigma_{I L, h}^{n+1 n+2} g_{L L}^{a, h h} \sigma_{L I, h}^{n+2 n+1} g_{I I}^{a, h e}$
from $\left(g_{R R}^{r} T_{R I}^{r} g_{I I}^{+-} T_{I R}^{a}\right)_{e e}^{n}$ :
5. $g_{R R}^{r, e e} \sigma_{R I, e}^{n n+1} g_{I I}^{r, e h} \sigma_{I L, h}^{n+1 n+2} g_{L L}^{r, h h} \sigma_{L I, h}^{n+2 n+1} g_{I I}^{+-, h e} \sigma_{I R, e}^{n+1 n}$
6. $g_{R R}^{r, e e} \sigma_{R I, e}^{n n+1} g_{I I}^{+-, e h} \sigma_{I L, h}^{n+1 n+2} g_{L L}^{a, h h} \sigma_{L I, h}^{n+2 n+1} g_{I I}^{a, h e} \sigma_{I R, e}^{n+1 n}$.

Terms 2 and 3 cancel. Like in the examples before for the numerical calculation we rest with products of four $g$-functions, again to be multiplied by $t_{L}^{2} t_{R}^{2}$ here. The argument is $\omega$ for $g_{R R}, \omega-A-C+2(n+1) B$ for $g_{L L}$ and $\omega-C+(n+1) B$ for $g_{I I}$. All Andreev reflections in these terms happen on the island. Having fixed the outer charge index to $n$ and the outer particle index to $e$ you could think that, for example, from $\left(g_{R R}^{r} T_{R L}^{r} g_{L L}^{+-} T_{L R}^{a}\right)_{e e}^{n}$ there could be another possible term, namely $g_{R R}^{r, e h} \sigma_{R I, h}^{n n-1} g_{I I}^{r, h h} \sigma_{I L, h}^{n-1 n} g_{L L}^{+-, h e} \sigma_{L I, e}^{n n+1} g_{I I}^{a, e e} \sigma_{I R, e}^{n+1}$. But writing 
(a)

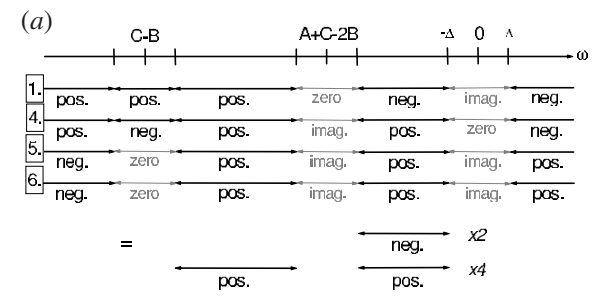

(c)

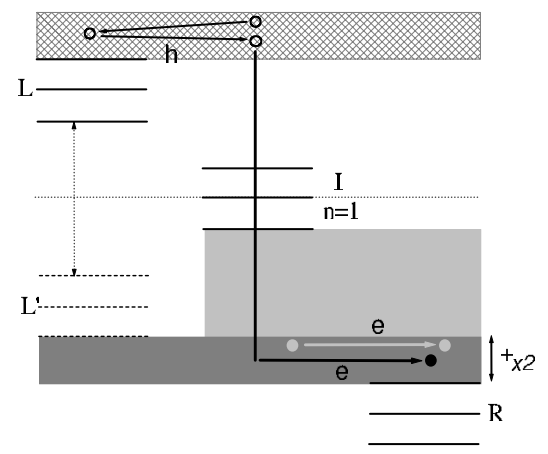

(b)

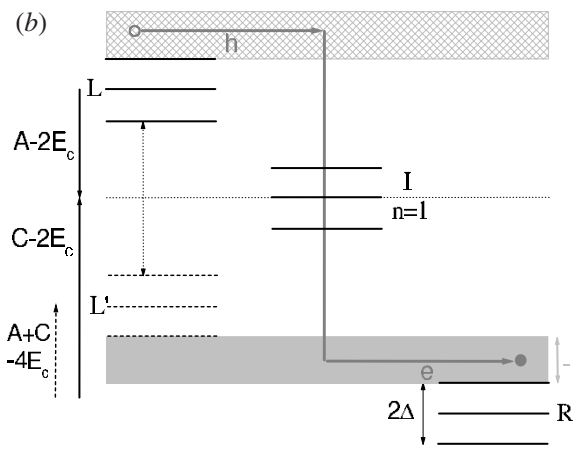

$(d)$

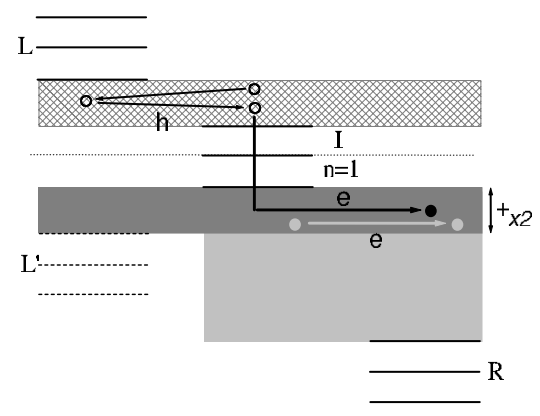

Figure 7. (a) Signs of products from terms (24). (b) Crossed AR in island state $n=1$ with electron transfer to R. (c) Interference of single electron transfer with that preceded by a hole backreflection to the left lead and AR on I in the same interval as crossed AR from $(b)$. (d) Another interval where the process from $(c)$ is possible. The image of the left gap mirrored at the island Fermi level determines interval boundaries here.

out phase factors the way we showed in (19) it is noted that the remaining phase with $\tau$ does not vanish. This $4 \sigma$-combination is not a dc contribution. Mirroring at the left Fermi level and then at the right-mirroring is what AR means in the energy-level diagram-cannot lead back to the initial energy level except for zero applied voltage. For the remaining four terms of (24) an overview on signs of their contributions in the different intervals is drawn in figure 7(a). Figures $7(b)-(d)$ give the interpretation in the energy-level diagram. Crossed AR in the island $n=1$ state, regarded as an example and contributing an electron transfer to $\mathrm{R}$ to decharge the island, is possible at all energy levels above the gap of $\mathrm{R}$ that mirrored at the $n=1$ island Fermi level come to lie above the gap of L (figure $7(b)$ ). This is the negative contribution from figure 7(a) (interest in the decharging process is an appropriate point of view here). However, there is a twice as large opposite contribution to the rate in that same interval from the single electron transfer interfering with an electron transfer preceded by the backreflection of a hole from the left lead (figure 7(c)). And there may be another interval, in which the latter process takes place (figure $7(d)$ ). These are the positive contributions from figure 7 $(a)$. (The interference process does not exist where the hole reflection would fall inside the gap left - even if no real state is required here - a property that can only be deduced from $g$-function plots. Energy considerations and level plots alone would not suggest this detail.) Again, the process sketched in figure $7(b)$ belongs to the kind associated with the bold-faced term in (23), those from figures $7(c)$ and $(d)$ to the bold-faced term in (22). In contrast to the direct lead-to-lead transport discussed earlier, crossed AR is more than counterbalanced 


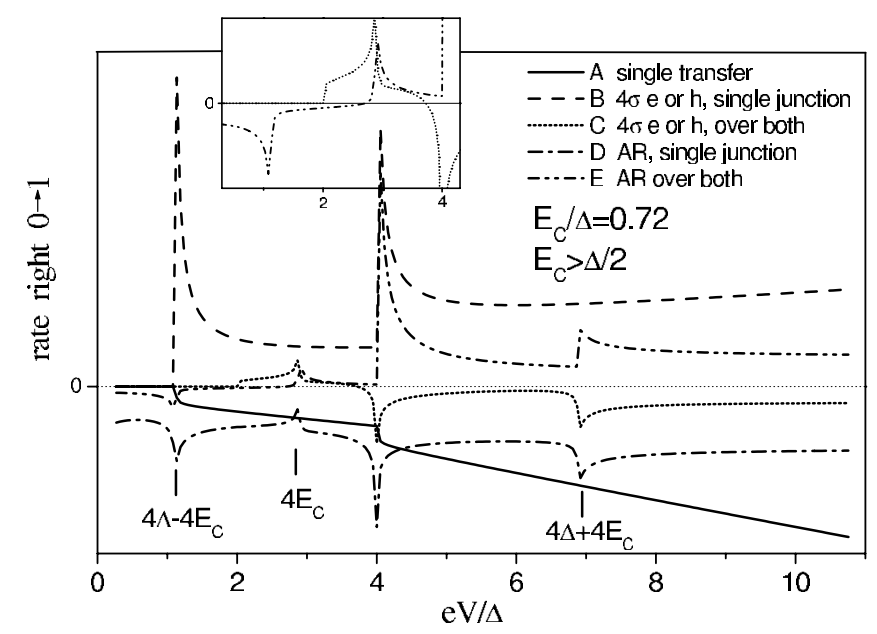

(a)

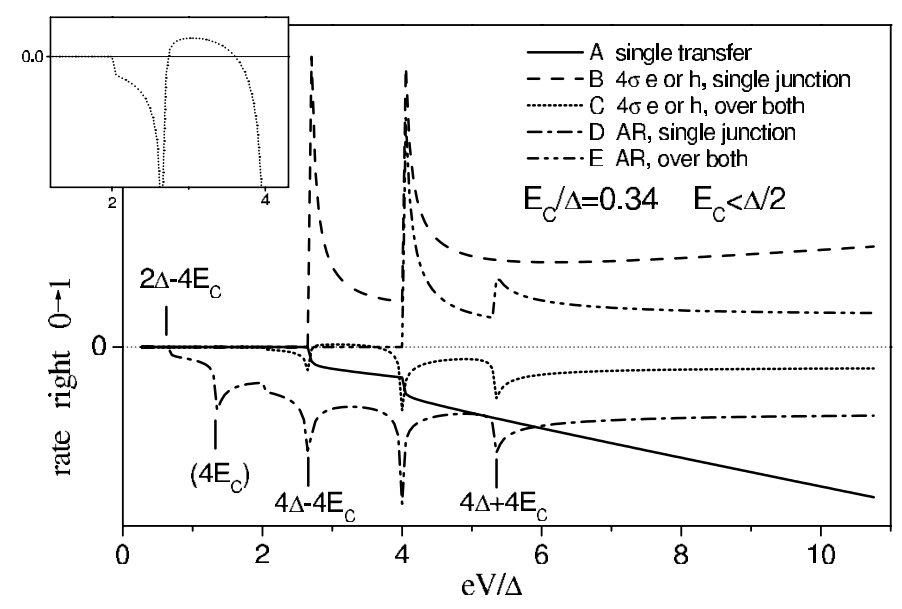

(b)

Figure 8. Qualitative calculation of low-order contributions to the $1 \rightarrow 0$ decharging rate across the right junction in a symmetric system with $\theta_{1}=\theta_{2}=0.5$ for two different ratios $E_{C}: \Delta$. Insets are magnifications of selected parts of some curves.

by an interference term of the same order. This interference term hindering decharging here could lower the current in the coherent as compared to the incoherent model, but we cannot expect any signs of crossed AR in current-voltage characteristics. For the symmetric case with the island $n=0$ state midway between $\mathrm{L}$ and $\mathrm{R}$ in potential, crossed $\mathrm{AR}$ in the $n=0$ state-which would contribute hole transfer from $\mathrm{R}$ to I to the $1 \rightarrow 0$ decharging rate across the right junction-is impossible. For the energy intervals, in which the processes sketched in figures $7(b)$ and $(c)$ take place, to exist, $E_{C}>\Delta / 2$ is mandatory.

In figure 8 we plot contributions to the rate for changing the island charge between 0 and 1 across the right junction arising from the low-order terms given here and in [10]. The right lead is held at $V$, the left lead at zero potential. For simplicity we apply no gate voltage and assume equal junction capacitances (onset thresholds as marked and singularity positions as expressed in simple multiples of $\Delta$ and $E_{C}$ only hold for this symmetric case). 
Also equal transmissions are chosen (unequal $t_{L}$ and $t_{R}$ would change the absolute values of the contributions, but not the positions of the peaks [11]). The potential of the neutral island state $n=0$ is situated midway between the lead Fermi levels. The $n=1$ state is $2 E_{C} / e$ above that level. (In the symmetric case with equal junction transmissions the main current contributions involve the island charge states nearest to zero.) From the potential landscape we thus expect that in stationary current flow the island is decharged across the right junction, that is electrons go off to the right lead. The Green's functions method does not add contributions order by order, the calculations displayed in figure 8 are made for purely analytical purposes (singularities in $g$-functions have been cut to finite values for simplicity). Single (A) electron transfers for $1 \rightarrow 0$ decharging start at $e V=4 \Delta-4 E_{C}$, hole transfers join at $4 \Delta$. Next higher-order pure electron or hole multiple reflections (B), see (18), have the same thresholds. Contribution (B) has opposite sign to (A). However, even without AR, the total $0 \rightarrow 1$ charging rate will be negative (island decharging), which is due to following higher orders having alternate signs. In contrast to the Green's functions method or analytic all-order summations [16] in approximations up to a finite order the onset singularities can never cancel correctly. The threshold for the $1 \rightarrow 0$ transition as part of an AR taking place in the right junction only (D) is $2 \Delta-4 E_{C}$, which in figure $8(a)$ even corresponds to a negative voltage. The contribution (C) from coherent transport over both junctions without $e$-conversion, see terms (20), indeed sets in at $2 \Delta$. However, only in figure $8(b)$ with the smaller of the two $E_{C}$ here do we get a negative rate corresponding to the desired direction of current flow. In figure $8(a)$ the interference parts are stronger than direct lead-to-lead transport. In the electron analog to figure 5 with the $n=1$ island state the $g$-products from the respective dark grey part can well give a larger contribution than those from all the light grey range between $\mathrm{L}$ and $\mathrm{R}$, even larger by more than the I-gap part from the holes in figure 5. Note further that an alike situation is also found in figure $8(b)$ between about $4 \Delta-4 E_{C}$ and $4 \Delta$. Only at about $4 \Delta$ does the lead-to-lead coupling via the 0 and 1 island states change into a definitive flow direction in any case. (The aforementioned direct lead-to-lead part in a total current-voltage characteristic of the two-junction series for low charging energy plays on more than just the 0 and 1 island states.) As contribution (E) from terms of the kind (24) in figure $8(b)$ we get the hole analog to figure $7(d)$ starting at $4 \Delta$ and the process as in figure $7(d)$ starting at $4 \Delta+4 E_{C}$. As discussed, this contribution hinders decharging in the coherent model and accordingly has positive sign here. The same two clear onsets are found in figure $8(a)$. However, there is a small positive contribution already setting in at $4 E_{C}$, and for lower voltages terms (24) even produce a negative one. The former is the process shown in figure $7(c)$. The latter is crossed AR, however, with the I $n=1$ Fermi level above that of $\mathrm{L}\left(V<4 E_{C}\right)$, which effectively allows AR inside the island's $n=1$ gap [10]. Unfortunately, crossed AR helping to decharge the island at such low voltages (peaked at $4 \Delta-4 E_{C}$ due to singularity matching) is of no use for establishing a stationary current all through the system as sufficiently effective up-charging processes - they also have to outweigh the 'wrong' part of the crossed AR through the left junction-require at least $e V=2 \Delta\left(4 \Delta-4 E_{C}<4 E_{C}\right.$ and $4 \Delta-4 E_{C}>2 \Delta$ is impossible at the same time). Our model does not at all regard spin polarization which might render crossed $\operatorname{AR}[17,18]$ much more interesting also in this system with the island changing in potential with charging.

\section{Frequency arguments and form of rate terms}

In terms without AR only three frequency arguments occur, one for each site, which will always be the same regardless of the number of reflections after which the particle comes back to that site. Anyone of the three can be taken as the integration variable, we can have 
$g_{R R}(\omega), g_{I I}(\omega)$ or $g_{L L}(\omega)$ as long as the choice is the same for all electron (hole) contributions. Each site having the same argument in all pure electron and in all pure hole contributions of an order contributing to the $n \rightarrow n+1$ island charging rate through a junction, is the only absolutely necessary condition for mathematical consistency. Terms with AR discussed so far only included one $g_{L L}$ and one $g_{R R}$ and the arguments of the two appearing $g_{I I}$ came out identical for (24). Higher-order MAR can contain different frequency arguments associated with the same site. In terms with AR a consistent choice of frequency arguments would, in principle, be unnecessary. They contain $g_{e h / h e}$ which become arbitrarily small for large negative and positive $\omega$. Thus with sufficiently wide boundaries there are no integral parts from half-open intervals that could be incorrectly counterbalanced. However, for the integration of these rather sharply peaked functions it seems important for numerical performance to have integrands which cancel in the complete outer half-open intervals up to the gap edge where individual contributions become singular. Besides that shifting argument sequences into the same patterns was essential for our analysis to show how different kinds and orders of transport processes are contained in the model. Nevertheless, there does not seem to lie any advantage in using rates in the form (17) with the negative terms, as their contributions cancel against others, anyway. One could use (10) caring to have $g_{R R}^{r}(\omega)$ in the first and in the last term and $g_{R R}^{+-}(\omega)$ in the middle term and preparing the three kinds of transfer functions $T_{L R}, T_{R R}$ and $T_{I R}$ in Fourier space with $\mathrm{R}$ for reference site [12]. (The reference site is the second of the two indices in representation (14). Using (15) without swapping $\mathrm{r}$ and a, $n$ - and site indices on $T$ in the integral compared to $T$ on the left side as well as without the $\dagger$-conjugation would make it the first.) For the trace it is of no importance that the $\sigma_{I R}$ at the end of the second term in (10) entered into $T_{R R}$ instead of being put in front as in (11). Thus in (10) from the product with the 1 from the parenthesis, $g_{R R}^{+-} T_{R R}^{a}$ is taken as an intensity. It looks awkward that in contrast to $g_{I I}^{+-}$- and $g_{L L}^{+-}$-parts with $g_{R R}^{+-}$the contribution with no interaction points on one half of the Keldysh contour (see figure 2) appears as a special term. Nevertheless, the following ansatz seems a promising idea, for which we rewrite (10) such that only $T_{R I / I R}$ and $T_{L I / I L}$ appear:

$$
\begin{aligned}
g_{R R}^{r} \underbrace{\sigma_{R I} g_{I I}^{r} T_{I L}^{r}}_{T_{R L}^{r}} g_{L L}^{+-} T_{L I}^{a} g_{I I}^{a} \sigma_{I R}+g_{R R}^{r} T_{R I}^{r} g_{I I}^{+-} T_{I R}^{a} \\
+g_{R R}^{+-} \underbrace{T_{R I}^{a} g_{I I}^{a} \sigma_{I R}}_{T_{R R}^{a}}+g_{R R}^{r} \underbrace{\sigma_{R I} g_{I I}^{r} T_{I R}^{r}}_{T_{R R}^{r}} g_{R R}^{+-} \underbrace{T_{R I}^{a} g_{I I}^{a} \sigma_{I R}}_{T_{R R}^{a}} .
\end{aligned}
$$

To construct these $T$-functions the two-step procedure from [11] is only needed for one kind of seed term. The functions can be used in rates for both junctions, none is calculated exclusively for one side ${ }^{4}$. As integration variable $\omega$, take the argument of $g_{L L}^{+-}$in the first, the one of $g_{R R}^{r}$ in the second and that of $g_{R R}^{+-}$in the last two terms in (25). Then Fourier representations (14)/(15) can be implied in a way to leave both $T$ in products with two of them of the same argument and furthermore only the need to establish $T_{I R}$ and $T_{I L}$ by recursion, because $T_{R I}$ and $T_{L I}$ are then obtained by complex conjugation. To deal with $T$-functions of a unique argument at a time is desirable to such a high degree, because even with all-order corrections the rate functions exhibit sharp peaks and an adapted integration procedure works off arguments in a not a priori determined sequence; storage of $T$ for equally spaced $\omega$ would be of no use. In contrast to (17) multiplication with single $\sigma$ at the end of terms as in (25) will complicate a calculation by requiring case differentiations for different components in $e h$-space. This has to do with the

4 Efficiency in setting up explicit code equations and optimizing computation time had indeed also been a motivation for this ansatz prior to discovering the expression with all alike structured terms (17). Because harder to debug and finally not giving different physics, we have not pursued this alternative option to the stage of having error-free codes for calculating $I V$-characteristics. From trials and arguments given in the analysis of rate terms in this paper we, however, strongly suspect that the compact form (17) should prove superior in numerical performance. 

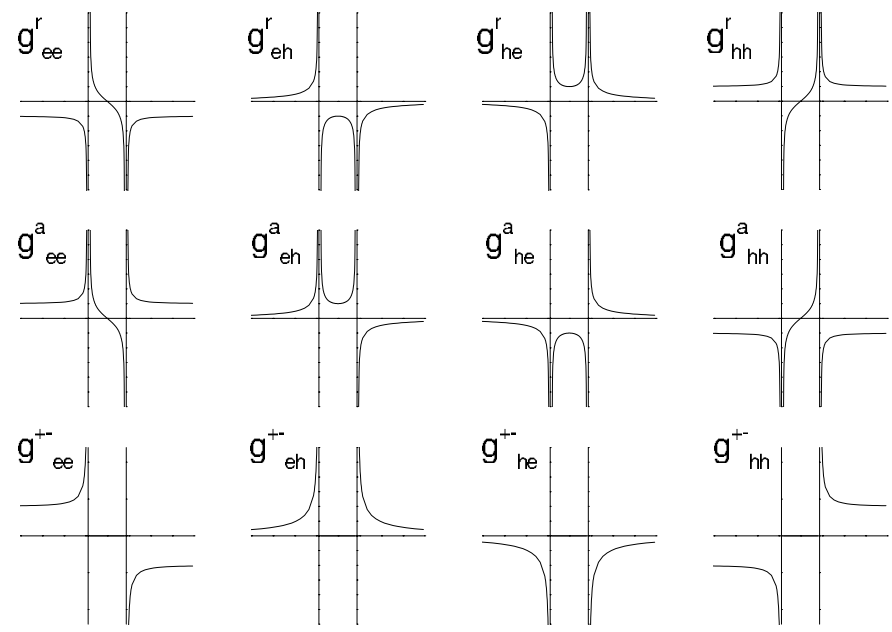

Figure 9. Basic $g$-functions.

choice of frequency arguments in the terms as well as the truncation of the range of allowed island charge states which both have to be consistent in the way all processes of the same order get included. Without going into further detail on these points, the following check reveals an inconsistency in the concept proposed with (25): we list the pure electron multiple reflection contributions with $4 \sigma$ and involving both leads. The island charge oscillates between $n$ and $n+1$. As in (19) the one fixed frequency argument determines the others.

(1) $g_{R R}^{r, e e} \sigma_{R I, e}^{n n+1} g_{I I}^{r, e e} \sigma_{I L, e}^{n+1 n} g_{L L}^{+-, e e} \sigma_{L I, e}^{a, e e} g_{I I}^{a, e e} \sigma_{I R, e}^{n+1 n}$

$$
\rightarrow g_{R R}^{r, e e}(\omega+C-A) g_{I I}^{r, e e}(\omega-A+(n+1) B) g_{L L}^{+-, e e}(\omega) g_{I I}^{a, e e}(\omega-A+(n+1) B)
$$

(2) $g_{R R}^{r, e e} \sigma_{R I, e}^{n n+1} g_{I I}^{r, e e} \sigma_{I L, e}^{n+1 n} g_{L L}^{r, e e} \sigma_{L I, e}^{n n+1} g_{I I}^{+-, e e} \sigma_{I R, e}^{n+1 n}$

$$
\rightarrow g_{R R}^{r, e e}(\omega) g_{I I}^{r, e e}(\omega-C+(n+1) B) g_{L L}^{r, e e}(\omega+A-C) g_{I I}^{+-, e e}(\omega-C+(n+1) B)
$$

(3) $g_{R R}^{r, e e} \sigma_{R I, e}^{n n+1} g_{I I}^{+-, e e} \sigma_{I L, e}^{n+1 n} g_{L L}^{a, e e} \sigma_{L I, e}^{n n+1} g_{I I}^{a, e e} \sigma_{I R, e}^{n+1 n}$

$$
\rightarrow g_{R R}^{r, e e}(\omega) g_{I I}^{+-, e e}(\omega-C+(n+1) B) g_{L L}^{a, e e}(\omega+A-C) g_{I I}^{a, e e}(\omega-C+(n+1) B)
$$

(4) $g_{R R}^{+-, e e} \sigma_{R I, e}^{n n+1} g_{I I}^{a, e e} \sigma_{I L, e}^{n+1 n} g_{L L}^{a, e e} \sigma_{L I, e}^{n n+1} g_{I I}^{a, e e} \sigma_{I R, e}^{n+1 n}$

$$
\rightarrow g_{R R}^{+-, e e}(\omega) g_{I I}^{a, e e}(\omega-C+(n+1) B) g_{L L}^{a, e e}(\omega+A-C) g_{I I}^{a, e e}(\omega-C+(n+1) B) \text {. }
$$

A factor of $t_{L}^{2} t_{R}^{2}$ has as usual been omitted on the right side in (26). Term 1 stems from the first term in (25), term 4 from the third and terms 2 and 3 from the second. The last term leaves no possibility of creating a product with only $4 \sigma$ containing $g_{L L}$ as well as $g_{R R}$. Drawing plots like in figure 4 would display the $2 \Delta$ - and surrounding intervals for term 1 shifted with respect to those for the other three terms. The outer half-open intervals would no longer cancel as they should for uniform integration boundaries. One could think about changing integration boundaries for the first term of (25), however, that is quite cumbersome (and for products with $e h$-conversion the shift would depend on the $n-, k$ - and $p$-indices of the $T$ involved). In conclusion, our considerations proved that although requiring the calculation of $T$-functions of more than two combinations of site indices, together with the rule to fix the reference argument 
with $g_{R R}$ for right-junction rates and with $g_{L L}$ for left junction rates, the form (17) of the rate formula is the most robust and the easiest in handling.

\section{Conclusions}

We have developed a model to calculate quantum transport in the normal as well as the superconducting state through two point-contacts in series with a bulky island between the two junctions, where processes in both junctions are coherently coupled together, however, the island in a classical way constantly changes its electrostatic potential with charging. The model merges a Green's functions technique taken from the treatment of the single junction with arbitrary transmission and a rate-equation method known to describe the single-electron transistor. This paper showed how to derive the formula for single-unit charging rates of the island through each junction in the situation with coherence maintained over the whole system. In analogy to the single junction these rates can be written as sums of 2-products of transfer functions. The established rate formula reveals that besides the direct coupling of a lead to the island there is an effective coupling of that lead to the remote reservoir which is the other lead. Technical details have been given concerning the precarious issue of keeping consistent energy reference levels in calculating transfer functions and charging rates. For analysis developing low-order contributions in transfer rates uncovers how intricately coherence and charging are linked. The direct lead-to-lead transport does not require intermediate states outside the superconductor gap on the island, Coulomb blockade may nevertheless suppress it. Crossed Andreev reflection is also contained in the calculation, however-within the premises of our model — does not produce additional features in current-voltage characteristics. Here we concentrated on the usage of Green's functions in charging rates. Current-voltage curves for the superconducting state are shown in [14]. Most important extensions to render the model more realistic and general will be the inclusion of Cooper-pair tunneling and the treatment of several transport channels per junction all coherently linked across the island.

\section{Appendix}

To help the interested reader reconstruct all interval analysis of the discussed low-order rate terms and eventually investigate others, we here schematically display all components of the $g$-functions. The vertical lines mark the $2 \Delta$-interval the respective function is centered in. Inside this interval all values are purely real, outside all are purely imaginary. Thus positive or negative values indicate signs of Re and Im, respectively. $g^{+-}$vanishes in the central interval and outside is just twice as large as $g^{r}$ or $g^{a}$.

\section{References}

[1] Grabert H and Devoret M 1992 Single Charge Tunneling, Coulomb Blockade Phenomena in Nanostructures (NATO ASI Series B vol 294) (New York: Plenum)

[2] Kouwenhoven L P, van der Vaart N C, Johnson A T, Kool W, Harmans C J P M, Williamson J G, Staring A A M and Foxon C T 1991 Z. Phys. B 85367

[3] Hadley P, Delvigne E, Visscher E H, Läteenmäki S and Mooij J E 1998 Phys. Rev. B 5815317

[4] Fitzgerald R J, Pohlen S L and Tinkham M 1998 Phys. Rev. B 57 R11073

[5] van den Brink A M, Schön G and Geerligs L J 1991 Phys. Rev. Lett. 673030

[6] Billangeon P M, Pierre F, Bouchiat H and Deblock R 2007 Phys. Rev. Lett. 98216802

[7] Bylander J, Duty T, Johansson G and Delsing P 2007 Phys. Rev. B 76020506

[8] Krans J M, Muller C J, Yanson I K, Govaert T C M, Hesper R and Ruitenbeek J M 1993 Phys. Rev. B 4814721 
[9] Scheer E, Agrait N, Cuevas J C, Levy Yeyati A, Ludoph B, Martin-Rodero A, Rubio-Bollinger G, van Ruitenbeek J M and Urbina C 1998 Nature 394154

[10] Schröter U and Scheer E 2006 Phys. Rev. B 74245301

[11] Schröter U and Scheer E 2008 J. Phys. A: Math. Theor. 41265202

[12] Schröter U 2008 Preprint arXiv:0802.3001

[13] Cuevas J C, Martin-Rodero A and Levy-Yeyati A 1996 Phys. Rev. B 547366

[14] Schröter U and Scheer E 2007 Phys. Rev. B 76205104

[15] D'Amato J L and Pastawski H M 1990 Phys. Rev. B 417411

[16] Averin D and Bardas A 1995 Phys. Rev. Lett. 751831

[17] Beckmann D, Weber H B and von Löhneysen H 2004 Phys. Rev. Lett. 93197003

[18] Deutscher G 2002 J. Supercond.: Incorporating Novel Magn. 1543 